\title{
On the irreducibility of symmetrizations of cross-characteristic representations of finite classical groups
}

\author{
Kay Magaard* \\ University of Birmingham \\ School of Mathematics, Watson Building \\ Edgbaston, Birmingham, B15 2TT, UK \\ Gerhard Röhrle ${ }^{\dagger}$ \\ Ruhr-Universität Bochum \\ Fakultät für Mathematik, Universitätstrasse 150 \\ Bochum D-44780 \\ Donna M. Testerman \\ Ecole Polytechnique Fédérale de Lausanne \\ Section de Mathématiques, MATHGEOM, Station 8 \\ CH-1015 Lausanne, Switzerland
}

November 19, 2018

\begin{abstract}
Let $W$ be a vector space over an algebraically closed field $k$. Let $H$ be a quasisimple group of Lie type of characteristic $p \neq \operatorname{char}(k)$ acting irreducibly on $W$. Suppose also that $G$ is a classical group with natural module $W$, chosen minimally with respect to containing the image of $H$ under the associated representation. We consider the question of when $H$ can act irreducibly on a $G$-constituent of $W^{\otimes e}$ and study its relationship to the maximal subgroup problem for finite classical groups.
\end{abstract}

\section{Introduction}

Let $C(V)$ be a finite classical group with natural module $V$. In his celebrated paper (Asc84), Aschbacher defined a collection $\mathcal{C}(C(V))$ of subgroups of $C(V)$, and proved that maximal subgroups of $C(V)$ which are not members of $\mathcal{C}(C(V))$ are necessarily of the form $N_{C(V)}(H)$, where $H$ is a quasisimple group acting absolutely irreducibly on $V$. Conversely, it is far from clear whether an absolutely irreducible $H$-module $V$ leads to a maximal subgroup of $C(V)$. For example, a possible obstruction to the maximality of $N_{C(V)}(H)$ could be the existence of a quasisimple group $G$ containing $H$ such that $N_{C(V)}(H) \subset N_{C(V)}(G)$. In this case we see that $V$ is also an absolutely irreducible $G$-module whose restriction to $H$ is irreducible. Thus we are naturally lead to consider branching problems in the representation theory of quasisimple groups. Our main theorem is the solution of one particular branching problem,

\footnotetext{
*k.magaard@bham.ac.uk

†'gerhard.roehrle@rub.de

‡donna.testerman@epfl.ch
} 
whose corollary is a contribution to the determination of the maximal subgroups of the finite classical groups.

Throughout we consider the following situation. Let $H$ be a perfect finite group of Lie type of characteristic $p$, and $W$ a vector space over an algebraically closed field $k$ of characteristic distinct from $p$. If $\rho: H \rightarrow \mathrm{GL}(W)$ is an irreducible representation of $H$, then the assumption that $H$ is perfect implies that $H \rho \subset \mathrm{SL}(W)$. The Frobenius-Schur indicator of $\rho$ determines whether or not $H \rho$ stabilizes a nondegenerate bilinear or quadratic form on $W$. If the indicator is zero, define $G:=\mathrm{SL}(W)$, else take the connected component $C$ of the stabilizer of the nondegenerate bilinear or quadratic form stabilized by $H \rho$ and define $G$ to be the simply connected version of the simple algebraic group determined by $C$. Let $V$ be a finite-dimensional irreducible $k G$-module. We consider the question of when the restriction of $V$ to $H \rho$ is reducible; it is reasonable to expect that in most cases this is indeed the case. Our main theorem shows that this is so if we assume that $H$ is classical and $W$ satisfies a certain technical hypothesis which we call $Q$-linear large. (The precise definitions of classical and $Q$-linear large are given below.)

Theorem 1. Let $p$ be a prime, $k$ an algebraically closed field of characteristic $\neq p$. Let $G$ be a simply connected simple algebraic group of classical type with natural module $W=k^{m}$. Let $H$ be a quasisimple finite classical group of type $X_{r}(q), q=p^{f}$, of untwisted Lie rank $r$ and characteristic $p$. Suppose that $W$ is a $Q$-linear large irreducible $k H$-module. If $H \subset G$, then for any restricted irreducible $k G$-module $V$ not isomorphic to $W$ or $W^{*}$, we have that $\left.V\right|_{H}$ is reducible. If $V$ is a twisted tensor product of restricted irreducible $k G$-modules, then $\left.V\right|_{H}$ is reducible unless $q \leq 3, H$ is not a central extension of $\operatorname{PSL}_{n}(q)$, and $V$ is a Frobenius twist of a module of the form $X \otimes Y^{\delta}$, where $X, Y \in\left\{W, W^{*}\right\}$, and $Y^{\delta}$ is a Frobenius twist of $Y$ such that $\left.X\right|_{H}$ and $\left.Y^{\delta}\right|_{H}$ are inequivalent $k H$-modules.

Our theorem has an immediate corollary.

Corollary 1.1. Let $p$ be a prime, $k$ an algebraically closed field of characteristic $\neq p$. Let $G$ be a classical group with natural module $V$ defined over $k$, and let $H$ be a quasisimple finite classical group of Lie type $X_{r}(q), q=p^{f}$, of untwisted Lie rank $r$ and characteristic p. Assume that $F$ is a Frobenius morphism of $G$ such that $H \subset G^{F}$ and $V^{F}$ is the natural module for $G^{F}$. If $H$ acts absolutely irreducibly on $V^{F}$ and there exists a classical simple algebraic group $G^{\prime}$ of characteristic char $(k)$ and a Frobenius morphism $F^{\prime}$ of $G^{\prime}$ such that $H \subset\left(G^{\prime}\right)^{F^{\prime}} \subset G^{F}$, then either the natural module $W$ of $G^{\prime}$ is not $Q$-linear large for $H$, or $q \leq 3, V$ is a Frobenius twist of a module of the form $X \otimes Y^{\delta}$, where $X, Y \in\left\{W, W^{*}\right\}$, and $Y^{\delta}$ is a Frobenius twist of $Y$ such that $\left.X\right|_{H}$ and $\left.Y^{\delta}\right|_{H}$ are inequivalent $k H$-modules.

Our corollary sheds more light on the set of maximal subgroups of finite classical groups. To see this suppose that we would like to study the maximal subgroups of a finite classical group $G^{F}$ whose natural module is $V^{F}$ as above. Suppose also that $H \subset G^{F}$ is of type $X_{r}(q)$, acts absolutely irreducibly on $V^{F}$, and that $(q, \operatorname{char}(k))=1$. What are the possible overgroups of $H$ in $G^{F}$ ? In the language of Aschbacher's reduction theorem ([Asc84]), a possible overgroup $K$ might be a member of one of the following families $\mathcal{C}_{2}, \mathcal{C}_{4}, \mathcal{C}_{6}, \mathcal{C}_{7}$, or $\mathcal{S}$.

The situation where $K$ is a member of $\mathcal{C}_{2}$, that is where $H$ acts imprimitively on $V^{F}$, is being investigated in $\mathrm{HHM}$. The situation where $K$ is a member of $\mathcal{C}_{4}$ was investigated by Magaard and Tiep [MT01]. The situation where $K$ is a member of $\mathcal{C}_{6}$ or $\mathcal{C}_{7}$ is being investigated in [MT] and independently in Bra].

The situation where $K$ is a member of $\mathcal{S}$ naturally subdivides according to the type of the simple group $K$. Evidently $V^{F}$ is an irreducible $K$-module whose restriction to $H$ is absolutely irreducible. Thus determining the maximal subgroups of finite classical groups requires the solution of certain branching rule problems. Branching rules for alternating and symmetric groups are an active area of current research. See for example JS92, KT04, BK01, BO98, KS02. The case where $K$ is a finite group of Lie type whose defining characteristic is 
equal to the defining characteristic of $H$ was investigated by Seitz in Sei90]. He shows that if $q>3$ then the possible pairs $(H, K)$ form four families of examples.

In this paper we discuss the case where $K=\left(G^{\prime}\right)^{F^{\prime}}$ is a member of $\mathcal{S}$, and is a finite classical group of Lie type over a field of characteristic equal to char $(k)$. Work of Malle Mal99, Magaard and Tiep [MT01, and Magaard, Malle and Tiep [MMT02 shows that there are families of examples. One such is obtained when $H=\mathrm{Sp}_{2 r}(3)$ and $V$ is the alternating or symmetric square of a Weil module $W$ of $H$. In this case $G=\operatorname{SL}(V)$ contains an irreducible subgroup $H$ with intermediate subgroup $\mathrm{SL}(W)$. Our main theorem shows that in quantifiable terms, such configurations are rare. To make this explicit, we now give the precise definition of $Q$-linear large.

Let $H$ be a finite classical group with natural module $N$ and let $P<H$ be the stabilizer of a singular 1-space of $N$. Then $P=Q L$, where $Q=O_{p}(P)$. We write $Q^{*}$ for the space $\operatorname{Hom}\left(Q /[Q, Q], k^{*}\right)$. For $\chi \in Q^{*}$ and a $k H$-module $W$, define $W_{\chi}:=\{w \in W \mid x w=$ $\chi(x) w$ for all $x \in Q\}$.

Definition 1.2. Let $P$ be as above. We say we are in the $Q$-linear case (or that $W$ is a $Q$-linear module for $H$ ) if either $Q$ is abelian or $Q$ is non-abelian and $[W, Q] \neq[W,[Q, Q]]$. We say we are in the $Q$-special case otherwise.

Remark. Note that we are in the $Q$-linear case when $H$ is linear or orthogonal. In addition, if we are in the $Q$-linear case, then there exists $\chi \in Q^{*}, \chi \neq 1$, with $W_{\chi} \neq 0$.

We now make a further case distinction, splitting the family of $Q$-linear modules into two subfamilies. For $\chi \in Q^{*}$, set $P_{\chi}:=\operatorname{Stab}_{P}\left(W_{\chi}\right)$; then $P_{\chi}=Q L_{\chi}$, where $L_{\chi}=\operatorname{Stab}_{L}\left(W_{\chi}\right)$. Write $L_{\chi}^{\infty}$ for the last term in the derived series of $L_{\chi}$.

Definition 1.3. Let $P$ be as above and assume we are in the $Q$-linear case.

(a) We say that we are in the $Q$-linear large case, or that $W$ is a $Q$-linear large module for $H$, if there exists $\chi \in Q^{*}, \chi \neq 1$, such that $\operatorname{soc}\left(\left.W_{\chi}\right|_{L_{\chi}^{\infty}}\right)$ has an irreducible summand $S$ of dimension greater than 1 .

(b) We say that we are in the $Q$-linear small case, or that $W$ is a $Q$-linear small module for $H$, if for all $\chi \in Q^{*}, \chi \neq 1$, all irreducible constituents of $\operatorname{soc}\left(\left.W_{\chi}\right|_{L_{\chi}^{\infty}}\right)$ are 1-dimensional.

Now returning to the example mentioned above, where $H=\operatorname{Sp}_{2 r}(3)$ and $W$ is a Weil module for $H$, we see that the statement of our main theorem fails if we drop the $Q$-linear large hypothesis. Indeed, the Weil modules are $Q$-special and hence not $Q$-linear large.

For finite classical groups $H$ of large untwisted Lie rank $r$ acting on an $\mathbb{F}_{q}$-vector space (or

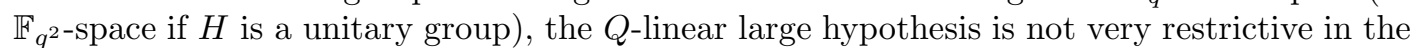
sense that the proportion of $Q$-linear large modules tends to 1 as $q$ and $r$ tend to infinity. To see this, note that the dimension of a $Q$-linear small module is bounded above by $\left[H: Q L_{\chi}^{\infty}\right]$ and the results of Guralnick, Magaard, Saxl, and Tiep in GMST02 show that the $Q$-special hypothesis implies that if $H$ is unitary, then $W$ is one of at most $(q+1)$ Weil modules, and that if $H$ is symplectic, then $W$ is either one of four Weil modules or of dimension bounded above $\frac{\left(q^{r}-1\right)\left(q^{r-1}-q\right)}{2(q+1)}$. In the latter case it follows by inspection that the module dimension is bounded by $\left[H: L_{\chi}^{\infty}\right]$. So in summary, if an $H$-module $W$ is not $Q$-linear large, then its dimension is bounded by $\left[H: Q L_{\chi}^{\infty}\right]$.

Now, according to Deligne-Lusztig theory, the (ordinary) Deligne-Lusztig character $\pm R_{T, \theta}$ is irreducible when $\theta$ is in general position. Moreover $\left|R_{T, \theta}(1)\right|=[H: T]_{p^{\prime}}$ is a polynomial function in $q$ of degree $\left|\Phi^{+}\right|$, where $\Phi^{+}$denotes the set of positive roots of the root system used to define $H$. For $H$ classical $\left|\Phi^{+}\right|$is a quadratic function in $r$ which shows that a module affording an irreducible $\pm R_{T, \theta}$ is $Q$-linear large, provided that $r \geq 4$.

Next we observe that each irreducible $\pm R_{T, \theta}$ corresponds uniquely to a regular semisimple class in $H^{*}$, the Deligne-Lusztig dual of $H$. When $r$ and $q$ tend to infinity then the proportion of regular semisimple classes of $H^{*}$ to the total number of conjugacy classes of $H$ tends to 1 . 
Now if $\ell=\operatorname{char}(k)$ and $\ell$ is a divisor of $|H|$, then the reduction of $\pm R_{T, \theta}$ modulo $\ell$ stays irreducible if the corresponding regular semisimple class in $H^{*}$ is represented by an element of order coprime to $\ell$. Again when $r$ and $q$ tend to infinity, then the proportion of regular semisimple $\ell^{\prime}$-classes of $H^{*}$ to the total number of $\ell^{\prime}$ conjugacy classes of $H$ tends to 1 , and thus the proportion of $Q$-linear large characters of $H$ tends to 1.

Our results are closely related to conjectures of Larsen and of Kollár and Larsen, both of which were proved by Guralnick and Tiep in GT05 respectively GT08. For a group $X$, an $X$ module $W=k^{m}$, and a positive integer $j$, define $M_{2 j}(X, W)$ to be $\operatorname{dim}\left(\operatorname{End}_{X}\left(W^{\otimes j}\right)\right)$. If $G$ is a classical group with natural module $W$, and $H$ is a finite subgroup of $G$, then Larsen conjectured that if $\operatorname{char}(k)=0$, then $M_{2 j}(H, W)>M_{2 j}(G, W)$ for some $j \leq 4$. Note that if $\operatorname{char}(k)=0$, then $M_{2 j}(H, W)=M_{2 j}(G, W)$ implies that every irreducible $G$-constituent of $W^{\otimes j}$ restricts irreducibly to $H$. The conjecture of Kollár and Larsen, now a theorem GT08, concerns the action of $H$ on $\operatorname{Sym}^{j}(W)$ and it roughly asserts that most of the time $H$ cannot act irreducibly for $j \geq 4$.

For the class of cross-characteristic $Q$-linear large modules of classical groups our results yield stronger versions of both conjectures.

Theorem 2. Let $H=X_{r}(q)$ be a quasisimple finite classical group, cross-characteristically embedded in a simple algebraic group $G$ of classical type, whose natural module is $W$. If $W$ is a $Q$-linear large $H$-module, then we have that no $k G$-constituent of $W^{\otimes a} \otimes\left(W^{*}\right)^{\otimes b}$ restricts irreducibly to $H$ for any $a+b=j \geq 2$. In particular, if $\operatorname{char}(k)=0$, then $M_{2 j}(H, W)>$ $M_{2 j}(G, W)$ for all $j \geq 2$.

We now make some remarks about how we prove our result. Assume that $W$ is a $Q$ linear large $k H$-module and that $V$ is a restricted irreducible module for $G$, the classical simple algebraic group with natural module $W$. Let $\lambda=\sum_{i=1}^{\ell} a_{i} \lambda_{i}$ be the highest weight of $V$, where $\lambda_{i}$ are the fundamental weights with respect to a fixed maximal torus and Borel subgroup of $G$ and $\ell=\operatorname{rank}(G)$. If $G \neq \mathrm{SL}(W)$ set $e(\lambda):=\sum_{i=1}^{\ell} a_{i} i$; for $G=\mathrm{SL}(W)$ set $e(\lambda):=\sum_{i=1}^{\ell} \min (i, \ell+1-i) a_{i}$. Let $B$ denote an upper bound for the dimension of an irreducible $k H$-module, as given in Sei90, Thm 2.1]. Using a result of Premet ([Pre87]), we are guaranteed that the weight spaces for certain weights subdominant to $\lambda$ are nontrivial. We show first that if $e(\lambda) \geq(\ell+1) / 2$, then there exists a subdominant weight $\mu$, occurring with nonzero multiplicity in $V$, such that the length of the Weyl group orbit of $\mu$ is greater than $B$. Thus from this point on we may assume that $e(\lambda)<(\ell+1) / 2$. Now for restricted modules $V$ for which $e(\lambda)<(\ell+1) / 2$, we produce an $L_{\chi}$-invariant submodule $V_{0}$, which by Frobenius reciprocity yields an upper bound of $\left[H: L_{\chi}\right] \operatorname{dim} V_{0}$ for $\operatorname{dim} V$. On the other hand, the $Q$-linear large hypothesis yields a lower bound for $\operatorname{dim} V$ of $\left(\operatorname{dim} V_{0}\right) 2^{e(\lambda)}\left(\begin{array}{c}\ell \\ e(\lambda)\end{array}\right)$. Comparing these bounds for $\operatorname{dim} V$ leads to a contradiction if $e(\lambda) \geq 3$. When $e(\lambda)=2$ we use MMT02 to conclude that none of the examples which occur there satisfy the $Q$-linear large hypothesis, proving the first part of our theorem. Finally, we use our result for restricted modules to deduce that if $V$ is a twisted tensor product, then all factors must be Frobenius twists of $W$ or its dual. Then we repeat the argument with the upper and lower bounds for $\operatorname{dim} V$ to conclude that the number of factors is at most 2 . Then we use the main theorem of MT01 to draw our conclusion.

It is natural to ask how difficult it is to remove the hypothesis that $W$ is $Q$-linear large. Firstly, there are examples of triples $(H, W, V)$, with $W$ a $Q$-linear small $k H$-module, and $V$ a $k G$-module on which $H$ acts irreducibly, both with $V$ restricted and with $V$ non-restricted. For example, if $H=\Omega_{7}(2) \cong \operatorname{Sp}_{6}(2)$ and $W$ is seven-dimensional and irreducible, then $W$ is not $Q$-linear large and the restricted $H$-module $V=\Lambda^{3}(W)$ is irreducible. Another example arises when $H=4_{1} \cdot \mathrm{PSL}_{3}(4)$. (We are using the notation of $\mathrm{CCN}+85$.) Here $H$ possesses exactly four inequivalent ordinary irreducible characters of degree 8, likewise also for all characteristics $s$ greater than 7 . If $s \equiv 2 \bmod 5$, then the standard Frobenius morphism $F$ induces a permutation of order two on the characters of degree 8 . So if $W$ is a module affording one of the characters of degree 8 , then $W \otimes W^{F}$ is an irreducible $H$-module. There are even 
infinite series of examples; if $H=\operatorname{Sp}_{2 r}(3)$, with $r \geq 2$ and $\operatorname{dim} W=\frac{q^{r} \pm 1}{2}$, then both $\Lambda^{2}(W)$ and $\operatorname{Sym}^{2}(W)$ are irreducible. We refer the reader to MMT02, MT01 for further examples of irreducible alternating, symmetric and tensor squares of non- $Q$-linear large modules. So we see that the $Q$-linear large hypothesis is necessary for some choices of $H$ and $W$.

Secondly, we remark that the initial parts of the analysis used here can be applied to the general case, i.e., to the $Q$-linear small case and to the non $Q$-linear case. However, establishing lower and upper bounds for $\operatorname{dim} V$ in terms of $e(\lambda)$ requires a detailed knowledge of the module $W$. Our expectation is that for large values of $q$ our results generalize completely, while for small values of $q$ we expect to obtain slightly weaker results. Note that one of the examples above shows that the irreducible module $V$ may occur in the 3 -fold tensor power of $W$. Nevertheless we expect that for every $q$ there exists an integer $e(q)$ such that for all restricted weights $\lambda$ with $e(\lambda)>e(q)$, the irreducible $k G$-module with highest weight $\lambda$ is a reducible $k H$-module. Our initial investigations into the non $Q$-linear large case lead us to believe that for $q$ large enough, $e(q) \leq 4$. The worst case encountered thus far is the case where $W$ is a Weil module for $H=\operatorname{Sp}_{2 r}(3)$, and our methods give $e(q) \leq 17$. The analysis of the non $Q$-linear large case is the subject of a forthcoming paper.

Finally we gratefully acknowledge a set of handwritten notes that was generously provided to us by Martin Liebeck and Gary Seitz, in which they outlined a strategy for proving our result. In addition, we thank the referee for a very careful reading of the paper and for suggesting various improvements and pointing out some essential corrections.

\section{Notation}

Fix a vector space $N$ of dimension $n$ over a finite field $K$ of characteristic $p$. Endow $N$ either with the trivial bilinear form, a nondegenerate alternating bilinear form, a nondegenerate quadratic form of Witt defect 0 or 1 , or a nondegenerate unitary form, respectively. If the form on $N$ is unitary, we assume that $K=\mathbb{F}_{q^{2}}$, and otherwise we take $K=\mathbb{F}_{q}$, where $q=p^{a}$, for $a \in \mathbb{N}$. Let $\operatorname{Isom}(N)$ denote the full group of isometries of $N$. We assume that $F^{*}(\operatorname{Isom}(N))$ is a quasisimple group. We recall that $F^{*}(X)$ denotes the generalized Fitting subgroup of a finite group $X$.

Definition 2.1. A quasisimple group $X$ is said to be a finite classical group of type $\mathbf{L}_{n}(q)$, $\mathbf{S}_{2 n}(q), \mathbf{O}_{n}(q)$, respectively $\mathbf{U}_{n}(q)$, provided $X$ is a central extension of the factor group $F^{*}(\operatorname{Isom}(N)) / Z\left(F^{*}(\operatorname{Isom}(N))\right)$, where $N$ is endowed as above with a trivial, alternating, quadratic, respectively unitary form.

By abuse of notation, we write $H=\mathbf{L}_{n}(q), \mathbf{S}_{2 n}(q)$, etc to mean that $H$ is of type $\mathbf{L}_{n}(q)$, $\mathbf{S}_{2 n}(q)$, etc. For example, when we write $H=\mathbf{S}_{4}(2)$, we mean that $H$ is either the alternating group on 6 letters or one of its cyclic central extensions. We use the notation $\boldsymbol{\Omega}_{2 m}^{+}(q)$ and $\boldsymbol{\Omega}_{2 m}^{-}(q)$ in place of $\mathbf{O}_{2 m}(q)$, when we need to distinguish between the two types of evendimensional orthogonal groups. The + type groups correspond to the case where the quadratic form has Witt index $m$ and the - type ones to the case where the quadratic form has Witt index $m-1$.

Remark. 1. Since $\mathbf{S}_{2 n}\left(2^{a}\right) \simeq\left(\mathbf{O}_{2 n+1}\left(2^{a}\right)\right)^{\prime}$, in the context of this paper, it is more convenient to regard symplectic groups over fields of characteristic 2 as orthogonal groups. Nevertheless, we allow ourselves to pass back and forth between these two points of view, since certain of the results taken from other sources view these groups as symplectic groups.

2. In addition, since certain of the groups in small dimension are isomorphic, we assume further that $n \geq 2$ for type $\mathbf{S}_{2 n}(q), n \geq 3$ for type $\mathbf{U}_{n}(q), n \geq 5$ for type $\mathbf{O}_{n}(q), n$ odd, and $n \geq 8$ for type $\mathbf{O}_{n}(q), n$ even. 
3. Finally, we note that we write $\operatorname{PSL}_{n}(q), \mathrm{U}_{n}(q), \operatorname{Sp}_{n}(q), \Omega_{n}^{ \pm}(q)$ and $\mathrm{O}_{n}(q)$, when we want to emphasize that we are not dealing with a central extension, but rather with a subgroup of the linear isometry group.

Definition 2.2. Each finite classical group has a rank, denoted $r$, defined in Table 1. In order to avoid duplication, we make various assumptions on $r$ as indicated.

\begin{tabular}{|l|l|l|}
\hline$H$ & $r$ & condition \\
\hline $\mathbf{L}_{n}(q)$ & $n-1$ & $r \geq 1$ \\
$\mathbf{U}_{n}(q)$ & $n-1$ & $r \geq 2$ \\
$\mathbf{S}_{2 n}(q)$ & $n$ & $r \geq 2$ \\
$\mathbf{O}_{2 n}(q)$ & $n$ & $r \geq 4$ \\
$\mathbf{O}_{2 n+1}(q)$ & $n$ & $r \geq 2, q$ even \\
& & $r \geq 3, q$ odd \\
\hline
\end{tabular}

Table 1: Rank $r$ of finite classical groups

We rely upon many basic structural results about quasisimple finite groups of Lie type, for example their orders, the index of a Borel subgroup, exceptional isomorphisms, as well as results on the sizes of orbits of vectors in the classical geometries. The basic resources for these results are Car89] and Gro02. $K$.

For a finite quasisimple group $K$, we write $K^{\infty}$ for the last term in the derived series of

\section{Some combinatorial lemmas}

We begin with a combinatorial lemma; the proof of (2) was kindly provided by Karen Collins.

Lemma 3.1. Let $j, m, a$ and $b$ be natural numbers such that $1 \leq j \leq m^{1 / 2}, a, b<\frac{m+1}{2}$ and $\frac{m}{2}<a+b \leq m$. Set $d=\left\lfloor\frac{m}{2}\right\rfloor$. The following inequalities hold.

(1) $\left(\begin{array}{c}m \\ j\end{array}\right)>m^{j / 2}$;

(2) $\frac{m !}{a ! b !(m-a-b) !} \geq\left(\begin{array}{c}m \\ d\end{array}\right)$.

Proof. For $j \leq m$, we note that $\frac{m-i}{j-i} \geq \frac{m}{j}$ for all $i \leq j-1$. Thus $\left(\begin{array}{c}m \\ j\end{array}\right) \geq\left(\frac{m}{j}\right)^{j}$, with strict inequality if $j<m$. The inequality of (1) follows directly.

For (2), first suppose $a+b=m$; since $a, b \leq \frac{m}{2}$, we have $m$ even, $d=m / 2$ and $a=\frac{m}{2}=b$. But then the inequality is clear.

So now assume $a+b<m$. First consider the case where $a \geq d$. Since $a+b<m, b<m-a$, so $\left(\begin{array}{c}m-a \\ b\end{array}\right)>1$. Since $a, d \leq m / 2$ and $a \geq d$, then $\left(\begin{array}{c}m \\ a\end{array}\right) \geq\left(\begin{array}{c}m \\ d\end{array}\right)$. So $m ! /(a ! b !(m-a-b) !)=$ $\left(\begin{array}{c}m \\ a\end{array}\right)\left(\begin{array}{c}m-a \\ b\end{array}\right)>\left(\begin{array}{c}m \\ d\end{array}\right)$ as claimed.

So we may now assume $a<d$, and by symmetry, $b<d$ as well. Recall that $d \leq m / 2<a+b$. We then have

$$
\frac{a ! b !(m-a-b) !}{d !(m-d) !}=\frac{a ! b !(m-a-b) !(d-b) !(a+b-d) !}{d !(m-d) !)(d-b) !(a+b-d) !}
$$

which is equal to

$$
\frac{a !}{(d-b) !(a+b-d) !} \cdot \frac{b !(d-b) !}{d !} \cdot \frac{(m-a-b) !(a+b-d) !}{(m-d) !}
$$


This latter equals $\left(\begin{array}{c}a \\ d-b\end{array}\right) /\left(\left(\begin{array}{c}d \\ d-b\end{array}\right)\left(\begin{array}{c}m-d \\ a+b-d\end{array}\right)\right)$.

Now $d>a$, so $\left(\begin{array}{c}d \\ d-b\end{array}\right)>\left(\begin{array}{c}a \\ d-b\end{array}\right)$ and $\left(\begin{array}{c}m-d \\ a+b-d\end{array}\right)>1$, since $m>a+b$. Hence the numerator is less than the denominator, and therefore, $a ! b !(m-a-b) !$ is less than $d !(m-d) !$, as desired.

Lemma 3.2. For $\ell \geq 2,\left(\begin{array}{c}\ell \\ \lfloor\ell / 2\rfloor\end{array}\right)>2^{\ell} /(\ell+1)$.

Proof. It suffices to note that $2^{\ell}$ is the sum of $\ell+1$ binomial coefficients, the largest of which is $\left(\begin{array}{c}\ell \\ \lfloor\ell / 2\rfloor\end{array}\right)$.

\section{Some weight theory}

Let $G$ denote a simple algebraic group of classical type defined over an algebraically closed field $k$ with $\operatorname{char}(k) \neq p$. That is, we fix a finite-dimensional vector space $W$ defined over $k$ and equip $W$ with either the trivial form or a nondegenerate alternating bilinear form, or a nondegenerate quadratic form and take $G$ to be $\left((\operatorname{Isom}(W))^{\prime}\right)^{\circ}$. By nondegenerate, we mean that the radical of the underlying bilinear form is trivial, with one exception: If $\operatorname{char}(k)=2$ and $W$ is an odd-dimensional orthogonal space, we say $W$ is "nondegenerate" provided the radical of the underlying bilinear form is a non-singular 1-space. By a slight (conventional) abuse of notation, we write $G=\mathrm{SL}(W), \mathrm{Sp}(W)$, respectively $\mathrm{SO}(W)$. When we wish to cover all cases at once, we simply refer to $G$ as $C l(W)$.

Let $T_{G}$ be a maximal torus of $G$, with character group $X\left(T_{G}\right)$, let $\Phi(G)$ denote the root system of $G$ relative to $T_{G}$, and take $\Pi(G)=\left\{\alpha_{1}, \alpha_{2}, \ldots, \alpha_{\ell}\right\}$ to be a fundamental system of $\Phi(G)$, and therefore $\operatorname{rank}(G)=\ell$. We label Dynkin diagrams as in Bou68. Let $\lambda_{i} \in X\left(T_{G}\right)$ denote the fundamental dominant weight corresponding to $\alpha_{i}$. Set $W_{G}=N_{G}\left(T_{G}\right) / T_{G}$, the Weyl group of $G$. For a dominant weight $\lambda \in X\left(T_{G}\right)$, we denote the rational irreducible $k G$-module with highest weight $\lambda$ by $V_{G}(\lambda)$. (All $k G$-modules considered are rational and hence we do not mention this in what follows.) Recall that a dominant weight is said to be restricted (and the corresponding irreducible module is referred to as restricted as well), if $\lambda=\sum_{i=1}^{\ell} a_{i} \lambda_{i}$ with $a_{i}<\operatorname{char}(k)$ for all $i$.

We require the following definition.

Definition 4.1. For a restricted dominant weight $\mu=\sum_{i=1}^{\ell} a_{i} \lambda_{i} \in X\left(T_{G}\right)$, set

$$
e(\mu)= \begin{cases}\sum_{i=1}^{\ell} i a_{i}, & \text { if } G \text { is of type } B_{\ell}, C_{\ell} \text { or } D_{\ell}, \\ \sum_{i=1}^{\ell} \min (i, \ell+1-i) a_{i}, & \text { if } G \text { is of type } A_{\ell} .\end{cases}
$$

In addition, we set

$$
\begin{gathered}
l_{\mu}= \begin{cases}0, & \text { if } a_{c}=0 \text { for all } c \leq \frac{\ell+1}{2} ; \\
\max \left\{c \mid 1 \leq c \leq \frac{\ell+1}{2} \text { and } a_{c} \neq 0\right\}, & \text { otherwise. }\end{cases} \\
r_{\mu}= \begin{cases}0, & \text { if } a_{c}=0 \text { for all } c>\frac{\ell+1}{2} ; \\
\max \left\{c \mid 1 \leq c<\frac{\ell+1}{2} \text { and } a_{\ell+1-c} \neq 0\right\}, & \text { otherwise. }\end{cases}
\end{gathered}
$$

We now prove a sequence of lemmas, whose aim is to establish a lower bound for $\operatorname{dim} V_{G}(\lambda)$ in terms of the rank $\ell$ of $G$ and $e(\lambda)$. The first result is taken from [Sei87].

Lemma 4.2. [Sei87, 1.10] Let $\mu$ be a dominant weight for $T_{G}$ and $W_{0}$ the subgroup of $W_{G}$ generated by those fundamental reflections corresponding to fundamental roots $\alpha \in \Pi(G)$ with $\langle\mu, \alpha\rangle=0$. Then $W_{0}$ is the full stabilizer of $\mu$ in $W_{G}$. In particular, the orbit of $\mu$ under the action of $W_{G}$ contains $\left|W_{G}: W_{0}\right|$ distinct weights. 
Lemma 4.3. Let $\mu \in X\left(T_{G}\right)$ be a restricted dominant weight, $\mu=\sum_{i=1}^{\ell} a_{i} \lambda_{i}$ and set $d_{0}=$ $\max \left\{d \mid a_{d} \neq 0\right\}$. Assume $a_{d_{0}}>1$ and in addition assume $d_{0}<\frac{\ell}{2}$ for $G$ of type $A_{\ell}, d_{0} \leq \ell-2$ for $G$ of type $B_{\ell}$ or $C_{\ell}$, and $d_{0} \leq \ell-3$ for $G$ of type $D_{\ell}$. Then the weight $\mu-\alpha_{d_{0}}$ is dominant; indeed,

$$
\mu-\alpha_{d_{0}}=\sum_{i=1}^{d_{0}-2} a_{i} \lambda_{i}+\left(a_{d_{0}-1}+1\right) \lambda_{d_{0}-1}+\left(a_{d_{0}}-2\right) \lambda_{d_{0}}+\lambda_{d_{0}+1} .
$$

Moreover $e\left(\mu-\alpha_{d_{0}}\right)=e(\mu)$ and $\mu-\alpha_{d_{0}}$ occurs with nonzero multiplicity in the irreducible $k G$-module with highest weight $\mu$.

Proof. Note that we interpret $\lambda_{0}$ as 0 in the above formula if $d_{0}=1$.

That $\mu-\alpha_{d_{0}}$ has the given form and is therefore a dominant weight is easily verified. Also Sei87, $1.5(\mathrm{c})]$ shows that $\mu-\alpha_{d_{0}}$ occurs with nonzero multiplicity in $V_{G}(\mu)$.

Now if $G$ is not of type $A_{\ell}$, that $e\left(\mu-\alpha_{d_{0}}\right)=e(\mu)$ follows directly from the definition. For $G$ of type $A_{\ell}$, since $d_{0}<\frac{\ell}{2}$, either $\ell$ is even and $d_{0} \leq \frac{\ell-2}{2}$ or $\ell$ is odd and $d_{0} \leq \frac{\ell-1}{2}$. A direct calculation establishes the equality $e\left(\mu-\alpha_{d_{0}}\right)=e(\mu)$.

Lemma 4.4. Let $\mu=\sum_{i<d} a_{i} \lambda_{i}+\lambda_{d} \in X\left(T_{G}\right)$ be a restricted dominant weight, $\mu \neq \lambda_{d}$, with $d<\frac{\ell}{2}$ if $G$ is of type $A_{\ell}$ and $d<\ell-2$ otherwise. Then there exists a weight $\nu \in X\left(T_{G}\right)$, subdominant to $\mu$, of the form $\nu=\sum_{j<d} b_{j} \lambda_{j}+\lambda_{d+1}$, with $e(\nu)=e(\mu)$. Moreover, the weight $\nu$ occurs with nonzero multiplicity in the irreducible $k G$-module with highest weight $\mu$.

Proof. Set $d_{0}=\max \left\{i<d \mid a_{i} \neq 0\right\}$. Set $\nu=\mu-\alpha_{d_{0}}-\alpha_{d_{0}+1}-\cdots-\alpha_{d}$. Then as in the above lemma one verifies that $\nu$ is dominant and satisfies $e(\nu)=e(\mu)$. Moreover, [Sei87, 8.6] shows that $\nu$ occurs with nonzero multiplicity in $V_{G}(\mu)$.

Lemma 4.5. Let $\mu=\sum_{i<d} a_{i} \lambda_{i}+\lambda_{d} \in X\left(T_{G}\right)$ be a restricted dominant weight, $\mu \neq \lambda_{d}$. Assume further that $d<\frac{\ell}{2}$ if $G$ is of type $A_{\ell}$, and $d<\ell-2$ otherwise. Set $m_{0}=\min \left\{e(\mu),\left\lfloor\frac{\ell}{2}\right\rfloor\right\}$, if $G$ is of type $A_{\ell}$, and set $m_{0}=\min \{e(\mu), \ell-3\}$, otherwise. Then for each $d \leq m \leq m_{0}$, there exists a weight $\nu_{m}$, subdominant to $\mu$ of the form $\nu_{m}=\sum_{i<d} b_{i} \lambda_{i}+\lambda_{m}$, with $e\left(\nu_{m}\right)=e(\mu)$. Moreover, each of the weights $\nu_{m}$ occurs with nonzero multiplicity in the irreducible $k G$-module with highest weight $\mu$.

Proof. We proceed by induction on $m$, noting that if $m=d$, we may take $\nu_{m}=\mu$. If $m=d+1$, the result follows from Lemma 4.4. For $m_{0} \geq m>d+1$, we suppose that there exists a weight $\nu_{m-1}=\sum_{j<d} b_{j} \lambda_{j}+\lambda_{m-1}$, subdominant to $\mu$ with $e\left(\nu_{m-1}\right)=e(\mu)$. Note that $\nu_{m-1} \neq \lambda_{m-1}$, else $e\left(\nu_{m-1}\right)=m-1=e(\mu)$, contradicting $e(\mu) \geq m_{0} \geq m$. Moreover $m-1<\frac{\ell}{2}$ if $G$ is of type $A_{\ell}$ and $m-1<\ell-2$ otherwise. So by Lemma 4.4 there exists a weight $\nu_{m}=\sum_{j<d} b_{j} \lambda_{j}+\lambda_{m}$, subdominant to $\nu_{m-1}$, and therefore subdominant to $\mu$, with $e\left(\nu_{m}\right)=e(\mu)$. The existence of the subdominant weight $\nu_{m}$ now follows by induction.

To see that the weight $\nu_{m}$ occurs with nonzero multiplicity in the irreducible $k G$-module $V_{G}(\mu)$, one must invoke the main result of [Pre87] and note that in all cases, the weights are obtained within a composition factor for a Levi subgroup of type $A$ whose root system has base $\left\{\alpha_{1}, \ldots, \alpha_{\left\lfloor\frac{\ell}{2}\right\rfloor}\right\}$ (respectively $\left\{\alpha_{1}, \ldots, \alpha_{\ell-2}\right\}$ ), for $G$ of type $A_{\ell}$ (resp. not of type $A_{\ell}$ ).

Lemma 4.6. Let $\mu \in X\left(T_{G}\right)$ be a restricted dominant weight with $r_{\mu}=0$; moreover assume $e(\mu)<\frac{\ell+2}{2}$ if $G$ is of type $A_{\ell}$, and $e(\mu)<\ell-2$ otherwise. Then the fundamental dominant weight $\lambda_{e(\mu)}$ is subdominant to $\mu$. Moreover, $\lambda_{e(\mu)}$ occurs with nonzero multiplicity in the irreducible $k G$-module with highest weight $\mu$.

Proof. Set $\mu=\sum_{i \leq m} a_{i} \lambda_{i}$, where $m \leq \frac{\ell}{2}$ and $a_{m} \neq 0$. If $\mu=\lambda_{m}$, then $e(\mu)=m$ and the result holds.

If $a_{m}>1$, then $2 m \leq e(\mu) \leq \frac{\ell+1}{2}$. Note that if $\ell<4$, then $\lambda=2 \lambda_{1}$ and $\lambda-\alpha_{1}$ is the desired subdominant weight. So we may assume $\ell \geq 4$, so we have $m<\frac{\ell-1}{2}$ if $G=A_{\ell}$ and 
$m \leq \ell-2$ otherwise; hence by Lemma 4.3, $\mu-\alpha_{m}$ is subdominant to $\mu$ and $e\left(\mu-\alpha_{m}\right)=e(\mu)$. Moreover, if $a_{m}>1$ and $\mu-\alpha_{m}$ is a fundamental dominant weight then $\mu=2 \lambda_{1}, \mu-\alpha_{1}=\lambda_{2}$ which is indeed $\lambda_{e(\mu)}$ as desired.

So we now suppose without loss of generality the existence of a subdominant weight $\nu$ satisfying $\nu=\sum_{i \leq m} b_{i} \lambda_{i}+\lambda_{d}$, where $m<d \leq \frac{\ell+2}{2}, \nu \neq \lambda_{d}$ and $e(\nu)=e(\mu)$.

Consider first the case where $G$ is of type $B_{\ell}, C_{\ell}$ or $D_{\ell}$. Here we have $e(\nu)<\ell-2$ and so $d<\ell-2$. By Lemma 4.5 there exists a subdominant weight $\nu_{r}$ of the form $\nu_{r}=\sum_{i<d} c_{i} \lambda_{i}+\lambda_{r}$, where $r=\min \{e(\nu), \ell-3\}$ and $e\left(\nu_{r}\right)=e(\nu)=e(\mu)$. But our assumption $e(\mu) \leq \ell-3$ implies that $r=e(\mu)$ and so $\nu_{r}=\lambda_{r}$ is the desired weight. Moreover, as in the previous proof, the weights are obtained via the action of a Levi subgroup of type $A$, and hence occur with nonzero multiplicity in the irreducible $k G$-module with highest weight $\mu$, by [Pre87.

We now turn to the case where $G$ is of type $A_{\ell}$. Here we have $e(\nu)=e(\mu)<\frac{\ell+2}{2}$. It suffices to establish the existence of the subdominant weight, as Pre87 then yields the result.

Suppose $\ell$ is odd and $e(\nu)=\frac{\ell+1}{2}$. Then $e(\nu)>d$ implies that $d<\frac{\ell}{2}$. Moreover, $r=\min \left\{e(\nu),\left\lfloor\frac{\ell}{2}\right\rfloor\right\}=\frac{\ell-1}{2}$ and so by Lemma 4.5. there exists a weight $\eta$, subdominant to $\nu$, of the form $\eta=\sum_{i<d} c_{i} \lambda_{i}+\lambda_{(\ell-1) / 2}$, with $e(\eta)=e(\nu)=\frac{\ell+1}{2}$. Since $e\left(\lambda_{(\ell-1) / 2}\right)=\frac{\ell-1}{2}$, $\eta \neq \lambda_{(\ell-1) / 2}$. So $c_{i} \neq 0$ for some $i<d$. Take $t$ maximal such that $c_{t} \neq 0$. Then $\rho=\eta-\left(\alpha_{t}+\right.$ $\left.\alpha_{t+1}+\cdots+\alpha_{(\ell-1) / 2}\right)$ is subdominant to $\eta$ and one checks that $e(\rho)=e(\eta)=e(\nu)=\frac{\ell+1}{2}$. But this then implies that $\rho=\lambda_{(\ell+1) / 2}$, satisfying the conclusion of the result.

Now suppose $\ell$ is even and $e(\nu)=\frac{\ell}{2}$. If $d=\frac{\ell+2}{2}$ or $\ell / 2$, then $e(\nu)>\ell / 2$. So we have that $d<\ell / 2$. Moreover, $r=\min \left\{e(\nu),\left\lfloor\frac{\ell}{2}\right\rfloor\right\}=e(\nu)=\frac{\ell}{2}$ and by Lemma 4.5, there exists a weight $\eta$, subdominant to $\nu$ of the form $\eta=\sum_{i<d} d_{i} \lambda_{i}+\lambda_{\ell / 2}$, with $e(\eta)=e(\mu)=\frac{\ell}{2}$. But this then implies that $\eta=\lambda_{\frac{\ell}{2}}$, which gives the result.

The two limiting cases having been handled, we can now assume that $e(\nu)<\frac{\ell}{2}$, so $d<\frac{\ell}{2}$ and $r=\min \left\{e(\nu),\left\lfloor\frac{\ell}{2}\right\rfloor\right\}=e(\nu)$, and by Lemma 4.5, there exists a weight $\nu_{r}$, subdominant to $\nu$ of the form $\nu_{r}=\sum_{i<d} d_{i} \lambda_{i}+\lambda_{r}$ with $e\left(\nu_{r}\right)=e(\nu)$. But $r=e(\nu)$ then gives $\nu_{r}=\lambda_{r}$, the desired subdominant fundamental weight.

We can now establish a bound for $\operatorname{dim} V_{G}(\lambda)$ in terms of $\operatorname{dim} W$ and $e(\lambda)$.

Proposition 4.7. Let $\lambda \in X\left(T_{G}\right)$ be a restricted dominant weight, set $e:=e(\lambda)$, and assume $e \leq \frac{\ell+1}{2}$ if $G$ is of type $A_{\ell}$, respectively $e \leq \ell-3$, otherwise. Then

$$
\operatorname{dim} V_{G}(\lambda) \geq\left(\begin{array}{c}
\ell+1 \\
e
\end{array}\right) \text {, if } G \text { is of type } A_{\ell},
$$

and

$$
\operatorname{dim} V_{G}(\lambda) \geq 2^{e}\left(\begin{array}{l}
\ell \\
e
\end{array}\right) \text {, if } G \text { is of type } B_{\ell}, C_{\ell} \text {, or } D_{\ell}
$$

Moreover, in all cases,

$$
\operatorname{dim} V_{G}(\lambda) \leq(\operatorname{dim} W)^{e}
$$

Proof. We first establish the upper bound. Recall that the irreducible $k G$-module with highest weight $\lambda_{i}$ occurs as a composition factor in $\Lambda^{i} W$, and therefore as a composition factor of $W^{\otimes i}$, for $i \leq \frac{\ell+1}{2}$, if $G$ is of type $A_{\ell}$, and for $i \leq \ell-3$ otherwise. For $G$ of type $A_{\ell}$, the $k G$-module of highest weight $\lambda_{i}, i>\frac{\ell+1}{2}$, occurs as a composition factor of $\Lambda^{\ell-i+1}\left(W^{*}\right)$, and therefore as a composition factor of $\left(W^{*}\right)^{\otimes(\ell-i+1)}$. Now if $G$ has type $B_{\ell}, C_{\ell}$ or $D_{\ell}$ and $e \leq \ell-3$, then $\lambda=\sum_{i=1}^{\ell-3} a_{i} \lambda_{i}$. Combining all of these remarks, we see that $V_{G}(\lambda)$ occurs as a composition factor of the $k G$-module $\otimes_{i=1}^{\ell}\left(\Lambda^{i} W\right)^{\otimes a_{i}}\left(\right.$ or $\otimes_{i=1}^{l_{\lambda}}\left(\Lambda^{i} W\right)^{\otimes a_{i}} \otimes \otimes_{i=1}^{r_{\lambda}}\left(\Lambda^{\ell-i+1} W^{*}\right) \otimes a_{\ell-i+1}$, where $l_{\lambda}$ and $r_{\lambda}$ are as in Definition 4.1). We then conclude that $\operatorname{dim} V_{G}(\lambda) \leq(\operatorname{dim} W)^{e}$, as desired.

Now turn to the lower bound. If $\lambda$ is a fundamental dominant weight, then Lemma 4.2 gives the desired lower bound. So we suppose $\lambda$ is not a fundamental dominant weight. If $G$ is of type $A_{\ell}$, write $\lambda=\lambda_{\ell}+\lambda_{r}$, where $\lambda_{\ell}=\sum_{i \leq l_{\lambda}} a_{i} \lambda_{i}$ and $\lambda_{r}=\sum_{i>l_{\lambda}} b_{i} \lambda_{i}$. 
If $G$ is of type $B_{\ell}, C_{\ell}$ or $D_{\ell}$, or if $G$ is of type $A_{\ell}$ and $\lambda=\lambda_{\ell}$ or $\lambda=\lambda_{r}$, then Lemma 4.5 implies that $\lambda_{e}$ is a subdominant weight occurring in the irreducible module $V_{G}(\lambda)$, (or in $\left.V_{G}(\lambda)^{*}\right)$ and hence we conclude as above. In the remaining case, when $G$ is of type $A_{\ell}$ and $\lambda \neq \lambda_{\ell}$ and $\lambda \neq \lambda_{r}$, we apply Lemma 4.5 to each of the weights $\lambda_{\ell}$ and $\lambda_{r}$. Note that $e=e\left(\lambda_{\ell}\right)+e\left(\lambda_{r}\right)$ and hence we find that $\lambda_{e\left(\lambda_{r}\right)}+\lambda_{e\left(\lambda_{\ell}\right)}$ is a subdominant weight occurring in the irreducible $k G$-module $V_{G}(\lambda)$. Here we use Lemmas $3.1(2)$ and 4.2 to see that $\operatorname{dim} V_{G}(\lambda) \geq \frac{(\ell+1) !}{e\left(\lambda_{\ell}\right) ! e\left(\lambda_{r}\right) !(\ell+1-e) !} \geq\left(\begin{array}{c}\ell+1 \\ e\end{array}\right)$.

Lemma 4.8. Let $G$ be of type $A_{\ell}$, and let $\lambda \in X\left(T_{G}\right)$ be a restricted dominant weight. If $e(\lambda)>(\ell+1) / 2$, then $\operatorname{dim} V_{G}(\lambda) \geq\left(\begin{array}{c}\ell+1 \\ \lfloor\ell / 2\rfloor\end{array}\right)$.

Proof. By [Pre87, all weights subdominant to $\lambda$ occur with nonzero multiplicity in $V_{G}(\lambda)$. It suffices to exhibit a weight such that the number of $W_{G}$-conjugates is at least $\left(\begin{array}{c}\ell+1 \\ \ell \ell / 2\rfloor\end{array}\right)$. Let $\lambda=\mu_{1}+\mu_{2}$, where $\mu_{1}=\sum_{i=1}^{\lfloor(\ell+1) / 2\rfloor} a_{i} \lambda_{i}$ and $\mu_{2}=\sum_{j=\lceil(\ell+2) / 2\rceil}^{\ell} b_{j} \lambda_{j}$.

If $e\left(\mu_{i}\right)<\frac{\ell+2}{2}$, for $i=1,2$, then we apply Lemma 4.6 to each of the weights $\mu_{i}, i=1,2$ to obtain a subdominant weight with nonzero coefficients of $\lambda_{e\left(\mu_{1}\right)}$ and $\lambda_{\ell+1-e\left(\mu_{2}\right)}$. Hence

$$
\operatorname{dim} V_{G}(\lambda) \geq \frac{(\ell+1) !}{e\left(\mu_{1}\right) ! e\left(\mu_{2}\right) !\left(\ell+1-e\left(\mu_{1}\right)-e\left(\mu_{2}\right)\right) !} .
$$

But since $e\left(\mu_{1}\right)+e\left(\mu_{2}\right)=e(\lambda)>\frac{\ell+1}{2}$, we may apply Lemma 3.1 with $m=\ell+1$ to obtain the desired inequality.

Suppose now that $e\left(\mu_{1}\right) \geq \frac{\ell+2}{2}$, and in particular, $\mu_{1}$ is not a fundamental dominant weight. Let $m$ be maximal such that $a_{m} \neq 0$. If $m \geq\lfloor\ell / 2\rfloor$, then the weight $\lambda$ has at least $\left(\begin{array}{c}\ell+1 \\ \ell / 2\rfloor\end{array}\right)$ conjugates, so we may assume $m<\ell / 2$. Now if $a_{m}=1$, we may use Lemma 4.5 to produce a subdominant weight with nonzero coefficient of $\lambda_{\lfloor\ell / 2\rfloor}$, which again gives the desired lower bound. So finally consider the case where $a_{m}>1$. Then

$$
\nu:=\lambda-\alpha_{m}=\sum_{i=1}^{m-2} a_{i} \lambda_{i}+\left(a_{m-1}+1\right) \lambda_{m-1}+\left(a_{m}-2\right) \lambda_{m}+\lambda_{m+1}+\mu_{2}
$$

is a subdominant weight with $e(\nu)=e(\lambda)$. Now $m+1 \leq \frac{\ell+1}{2}$; if $m+1 \geq \ell / 2$, there are at least $\left(\begin{array}{c}\ell+1 \\ \ell / 2\rfloor\end{array}\right)$ distinct conjugates of $\nu$ and if $m+1<\ell / 2$, we argue as above.

Finally, we must consider the case where $e\left(\mu_{2}\right) \geq \frac{\ell+2}{2}$. But this is completely analogous.

Lemma 4.9. Let $G$ be of type $B_{\ell}, C_{\ell}$ or $D_{\ell}$, with $\ell \geq 3$. Let $\lambda \in X\left(T_{G}\right)$ be a restricted dominant weight, $\lambda=\sum_{i=1}^{\ell} a_{i} \lambda_{i}$. Set $e:=e(\lambda)$. The following hold:

a) If $r_{\lambda} \neq 0$, then $\operatorname{dim} V_{G}(\lambda) \geq 2^{\ell-1}$.

b) If $r_{\lambda}=0$, and $e \leq(\ell+1) / 2$, then $\operatorname{dim} V_{G}(\lambda) \geq\left(\begin{array}{l}\ell \\ e\end{array}\right)$. If moreover $\ell \geq 7$, then $\operatorname{dim} V_{G}(\lambda) \geq$ $2^{e}\left(\begin{array}{l}\ell \\ e\end{array}\right)$.

c) If $r_{\lambda}=0$ and $e>(\ell+1) / 2$, then $\operatorname{dim} V_{G}(\lambda) \geq 2^{\ell-1}$.

Proof. For (a), we first note that if $a_{\ell} \neq 0$, or if $a_{\ell}+a_{\ell-1} \neq 0$ when $G=D_{\ell}$, then the lower bound follows directly from Lemma 4.2. So now assume $a_{\ell}=0$ and in addition $a_{\ell-1}=0$ if $G=D_{\ell}$. Again applying Lemma 4.2, we have $\operatorname{dim} V_{G}(\lambda) \geq 2^{\ell-r_{\lambda}+1}\left(\begin{array}{c}\ell \\ r_{\lambda}-1\end{array}\right)$. Now since $r_{\lambda} \leq \ell / 2$, we have that $\left(\begin{array}{c}\ell \\ r_{\lambda}-1\end{array}\right) \geq 2^{r_{\lambda}-1}$ and so $\operatorname{dim} V \geq 2^{\ell}$.

The bound of (b) follows from Proposition 4.7 as long as $\frac{\ell+1}{2} \leq \ell-3$. If $\ell=3,4$ or 5 , so $e \leq 2,2,3$ respectively, then the minimal dimension of an irreducible $k G$-module exceeds 
$\left(\begin{array}{c}\ell \\ e(\lambda)\end{array}\right)$. If $\ell=6$ and so $e \leq 3$, then it is a direct check to see that there exists a subdominant weight whose orbit under the Weyl group has length exceeding $\left(\begin{array}{c}\ell \\ e(\lambda)\end{array}\right)$.

Finally, for (c) assume $r_{\lambda}=0$ and $e(\lambda)>\frac{\ell+1}{2}$. We can argue exactly as in the proof of the previous lemma (in the case where $e\left(\mu_{1}\right) \geq \frac{\ell+2}{2}$ ) to produce a subdominant weight with $r_{\lambda} \neq 0$ and then use (a).

\section{$5 \quad$ Preliminary analysis}

Let $H=H_{r}(q)$ be a quasisimple finite classical group in characteristic $p$, let $k$ be an algebraically closed field of characteristic different from $p$. Let $W$ be a nontrivial $k H$-module. Let $G$ be the smallest classical group containing the image of the corresponding representation of $H$. We maintain the notation established in Sections 1 and 2, In particular, $P<H$ is the stabilizer of a singular 1-space of $N$, the natural module for $H$, and $P=Q L$, where $Q=O_{p}(P)$. We let $\chi \in Q^{*}=\operatorname{Hom}\left(Q /[Q, Q], k^{*}\right)$ and set $W_{\chi}=\{w \in W \mid x w=\chi(x) w$ for all $x \in Q\}$. Let $P_{\chi}:=\operatorname{Stab}_{P}\left(W_{\chi}\right)$; then $P_{\chi}=Q L_{\chi}$, where $L_{\chi}=\operatorname{Stab}_{L}\left(W_{\chi}\right)$. Write $L_{\chi}^{\infty}$ for the last term in the derived series of $L_{\chi}$.

It is useful to collect some information about the structure of $P, L_{\chi}$ and the orbit sizes of $L$ acting on $Q^{*}$. We record this information in Table 2 ,

Remark. 1) The $L$-orbit of singular vectors in $Q^{*}$ corresponds precisely to the cases where the stabilizer $L_{\chi}^{\prime}$ satisfies $O_{p}\left(L_{\chi}^{\prime}\right) \neq 1$.

2) When $H=\mathbf{U}_{r+1}(q)$, there are exactly $q-1$ distinct $L^{\prime}$-orbits of nonsingular vectors, all of which are fused by the action of the central torus of $L$.

3) When $H=\mathbf{O}_{n}(q)$, the situation is more complicated. When $p \neq 2$, or $p=2$ and $H$ is of type $\mathbf{O}_{2 r+1}(q)$, then there are $2 L^{\prime}$-orbits of nonsingular 1 -spaces which implies that there are $2 L$-orbits of nonsingular vectors on $Q$ (the natural module for $L^{\prime}$ ), whereas there is a unique $L^{\prime}$-orbit on nonsingular 1 spaces when $p=2$ and $H$ is of type $\boldsymbol{\Omega}_{2 r}^{ \pm}(q)$ leading to a single $L$-orbit on $Q$.

Lemma 5.1. Let $H$ and $W$ satisfy the $Q$-linear large hypotheses. Then one of the following holds.

a) $H=\mathbf{L}_{4}(q)$ and $q \geq 4$;

b) $H=\mathbf{L}_{r+1}(q)$ and $r \geq 4$;

c) $H=\mathbf{U}_{5}(q)$ and $q \geq 4$;

d) $H=\mathbf{U}_{6}(q)$ and $q \geq 3$;

e) $H=\mathbf{U}_{r+1}(q)$ and $r \geq 6$;

f) $H=\mathbf{S}_{6}(q)$ and $q \geq 5$;

g) $H=\mathbf{S}_{2 r}(q)$ and $r \geq 4$;

h) $H=\mathbf{O}_{2 r+1}(q)$ and $r \geq 3$;

i) $H=\boldsymbol{\Omega}_{2 r}^{ \pm}(q)$ and $r \geq 4$.

Proof. Note that the $Q$-linear large hypothesis implies that $L_{\chi}$ is nonsolvable. Relying upon the information in Table 2 it is a direct check to see that the only finite quasisimple classical groups omitted are those for which $L_{\chi}$ is solvable. 


\begin{tabular}{|c|c|c|c|c|}
\hline$H$ & $Q$ & $L^{\prime}$ & generic structure of $L_{\chi}$ & {$\left[L: L_{\chi}\right]$} \\
\hline $\mathbf{L}_{r+1}(q)$ & $q^{r}$ & $\mathbf{L}_{r}(q)$ & $q^{r-1}: \mathbf{L}_{r-1}(q)$ & $q^{r}-1$ \\
\hline $\mathbf{U}_{r+1}(q)$ & $q^{1+2(r-1)}$ & $\mathbf{U}_{r-1}(q)$ & $\begin{array}{l}q^{1+2(r-3)}: \mathbf{U}_{r-3}(q) \\
\mathbf{U}_{r-2}(q)\end{array}$ & $\begin{array}{l}\left(q^{r-1}+(-1)^{r}\right)\left(q^{r-2}-(-1)^{r}\right) \\
(q-1) q^{r-2}\left(q^{r-1}-(-1)^{r-1}\right)\end{array}$ \\
\hline $\begin{array}{l}\mathbf{S}_{2 r}(q) \\
q \text { odd }\end{array}$ & $q^{1+2(r-1)}$ & $\mathbf{S}_{2(r-1)}(q)$ & $q^{1+2(r-2)}: \mathbf{S}_{2(r-2)}(q)$ & $q^{2(r-1)}-1$ \\
\hline $\mathbf{O}_{2 r+1}(q)$ & $q^{2 r-1}$ & $\mathbf{O}_{2 r-1}(q)$ & $\begin{array}{l}q^{2 r-3}: \mathbf{O}_{2 r-3}(q) \\
\boldsymbol{\Omega}_{2 r-2}^{+}(q) \\
\boldsymbol{\Omega}_{2 r-2}^{-}(q)\end{array}$ & $\begin{array}{l}q^{2 r-2}-1 \\
\frac{(q-1)}{2}\left(q^{2 r-2}+q^{r-1}\right) \\
\frac{(q-1)}{2}\left(q^{2 r-2}-q^{r-1}\right)\end{array}$ \\
\hline $\boldsymbol{\Omega}_{2 r}^{+}(q)$ & $q^{2 r-2}$ & $\boldsymbol{\Omega}_{2 r-2}^{+}(q)$ & $\begin{array}{l}q^{2 r-4}: \boldsymbol{\Omega}_{2 r-4}^{+}(q) \\
\mathbf{O}_{2 r-3}(q)\end{array}$ & $\begin{array}{l}q^{2 r-3}+q^{r-1}-q^{r-2}-1 \\
\left(\frac{q-1}{2-\delta_{2, p}}\right)\left(q^{2 r-3}-q^{r-2}\right)\end{array}$ \\
\hline $\boldsymbol{\Omega}_{2 r}^{-}(q)$ & $q^{2 r-2}$ & $\mathbf{\Omega}_{2 r-2}^{-}(q)$ & $\begin{array}{l}q^{2 r-4}: \boldsymbol{\Omega}_{2 r-4}^{-}(q) \\
\mathbf{O}_{2 r-3}(q)\end{array}$ & $\begin{array}{l}q^{2 r-3}-q^{r-1}+q^{r-2}-1 \\
\left(\frac{q-1}{2-\delta_{2, p}}\right)\left(q^{2 r-3}+q^{r-2}\right)\end{array}$ \\
\hline
\end{tabular}

Table 2: Orbit size and structure

We record in Table 3] the Landazuri-Seitz [LS74], Seitz-Zalesski [SZ93], and Guralnick-Tiep GT99] bounds for the minimal dimension of an irreducible cross-characteristic representation of $H$, noted $b_{1}$, for each of the classical groups $H$. In addition, we provide a lower bound of a simpler ( $q$-power) form. In the final column, we record an upper bound $B$ for the dimension of such a representation, deduced from [Sei90, Thm. 2.2].

Hence we have

Lemma 5.2. Let $U$ be a nontrivial irreducible $k H$-module. Then $b_{1} \leq \operatorname{dim} U \leq B$.

In addition, we now establish a better lower bound for $W$, given that we are working under the assumption that $H$ and $W$ satisfy the $Q$-linear large hypothesis.

Proposition 5.3. Let $H$ and $W$ satisfy the $Q$-linear large hypothesis. Assume moreover that $O^{p}\left(L_{\chi}^{\infty}\right)$ is not one of the exceptions in Table [3. Then $\operatorname{dim} W \geq b_{2}$, where $b_{2}$ is as recorded in Table 4 .

Proof. Since $W=C_{W}(Q) \oplus\left(\sum_{\chi \in Q^{*}, \chi \neq 1} W_{\chi}\right)$, and there exists $\chi \neq 1$ such that $\operatorname{soc}\left(\left.W_{\chi}\right|_{L_{\chi}^{\infty}}\right)$ 


\begin{tabular}{|c|c|c|c|}
\hline$H$ & $r$ & $b_{1}$ & $B$ \\
\hline $\mathbf{L}_{2}(q)$ & & $\frac{q-1}{\operatorname{gcd}(q-1,2)}$ & $q+1$ \\
\hline $\mathbf{L}_{r+1}(q)$ & $r \geq 2$ & $\frac{q^{r+1}-1}{q-1}-2>q^{r}+q^{r-1}$ & $q^{\frac{r(r+3)}{2}}$ \\
\hline \multirow[t]{2}{*}{$\mathbf{U}_{r+1}(q)$} & $r \geq 2$ even & $q\left(q^{r}-1\right) /(q+1)>q^{r}-q^{r-1}$ & $q^{\frac{r(r+3)}{2}}$ \\
\hline & $r \geq 3$ odd & $\left(q^{r+1}-1\right) /(q+1)>q^{r}-q^{r-1}$ & $q^{\frac{r(r+3)}{2}}$ \\
\hline $\mathbf{S}_{2 r}(q)$ & $r \geq 2$ & $\left(q^{r}-1\right) / 2>q^{r-1}$ & $q^{r^{2}+r}$ \\
\hline $\begin{array}{l}\mathbf{O}_{2 r+1}(q) \\
q \text { odd }, q \neq 3\end{array}$ & $r \geq 3$ & $\frac{q^{2 r}-1}{q^{2}-1}-r>q^{2 r-2}$ & $q^{r^{2}+r}$ \\
\hline$q=3$ & $r \geq 3$ & $\frac{3^{2 r}-1}{3^{2}-1}-\frac{3^{r}-1}{2}>q^{2 r-2}$ & $q^{r^{2}+r}$ \\
\hline$q$ even & $r \geq 2$ & $\frac{q\left(q^{r}-1\right)\left(q^{r-1}-1\right)}{2(q+1)} \geq q^{2 r-3}$ & $q^{r^{2}+r}$ \\
\hline $\begin{array}{l}\boldsymbol{\Omega}_{2 r}^{+}(q) \\
q \neq 2,3\end{array}$ & $r \geq 4$ & $\frac{q\left(q^{2 r-2}-1\right)}{q^{2}-1}+q^{r-1}-r>q^{2 r-3}$ & $q^{r^{2}}$ \\
\hline$q=2,3$ & & $\frac{q\left(q^{2 r-2}-1\right)}{q^{2}-1}-\frac{q^{r-1}-1}{q-1}-7 \delta_{2, q}>q^{2 r-3}$ & $q^{r^{2}}$ \\
\hline $\boldsymbol{\Omega}_{2 r}^{-}(q)$ & $r \geq 4$ & $\frac{q\left(q^{2 r-2}-1\right)}{q^{2}-1}-q^{r-1}-r+2>q^{2 r-3}$ & $q^{r^{2}}$ \\
\hline $\begin{array}{l}\text { Exceptions } \\
b_{1}\left(\mathbf{L}_{2}(4)\right)=2 ; \\
b_{1}\left(\mathbf{L}_{4}(3)\right)=26 ; \\
b_{1}\left(\varnothing_{7}(3)\right)=27\end{array}$ & $\begin{array}{l}b_{1}\left(\mathbf{L}_{2}(9)\right)=3 \\
b_{1}\left(\mathbf{O}_{5}(2)\right)=2\end{array}$ & $\begin{array}{l}b_{1}\left(\mathbf{L}_{3}(2)\right)=2 ; \quad b_{1}\left(\mathbf{L}_{3}(4)\right)=4 \\
b_{1}\left(\mathbf{U}_{4}(2)\right)=4 ; \quad b_{1}\left(\mathbf{U}_{4}(3)\right)=6\end{array}$ & $\begin{array}{l}b_{1}\left(\mathbf{L}_{4}(2)\right)=7 \\
b_{1}\left(\boldsymbol{\Omega}_{8}^{+}(2)\right)=8\end{array}$ \\
\hline
\end{tabular}

Table 3: Definition of the bounds $b_{1}$ and $B$

has an irreducible $L_{\chi}^{\infty}$-submodule $S$ of dimension greater than 1, we may obtain a lower bound for $\operatorname{dim} W$ as the product of the lower bound for a nontrivial irreducible $k L_{\chi}^{\infty}$-module, as given in Table 3, and the size of a minimal orbit of $L$ acting on $Q^{*}$, that is the minimal value for $\left[L: L_{\chi}\right]$ as in Table 2,

Remark 5.4. The groups for which some $O^{p}\left(L_{\chi}^{\infty}\right)$ is one of the exceptions in Table 3 , and which are therefore not covered by Proposition 5.3, are as follows:

(i) $H=\mathbf{L}_{4}(q), q=4,9$; (ii) $H=\mathbf{L}_{5}(q), q=2$, 4; (iii) $H=\mathbf{L}_{6}(q), q=2,3$;

(iv) $H=\mathbf{U}_{r+1}(q), r=4,5, q=4,9$; (v) $H=\mathbf{U}_{r+1}(q), r=6,7, q=2,3$; (vi) $H=\mathbf{S}_{6}(9)$; 


\begin{tabular}{|c|c|c|}
\hline$H$ & & $b_{2}$ \\
\hline $\mathbf{L}_{4}(q)$ & & $\frac{q-1}{\operatorname{gcd}(q-1,2)}\left(q^{3}-1\right)$ \\
\hline $\mathbf{L}_{r+1}(q)$ & $r \geq 4$ & $\left(\frac{q^{r-1}-1}{q-1}-2\right)\left(q^{r}-1\right)$ \\
\hline $\mathbf{U}_{5}(q)$ & $q \neq 4,9$ & $\frac{q-1}{\operatorname{gcd}(q-1,2)}(q-1) q^{2}\left(q^{3}+1\right)$ \\
\hline $\mathbf{U}_{6}(q)$ & $q \neq 4,9$ & $\frac{q-1}{\operatorname{gcd}(q-1,2)}\left(q^{7}+q^{4}-q^{3}-1\right)$ \\
\hline $\mathbf{U}_{r+1}(q)$ & $\begin{array}{l}r \geq 6 \\
r \text { even }\end{array}$ & $\frac{q^{r-3}-q}{q+1}\left(q^{2 r-3}-q^{r-1}+q^{r-2}-1\right)$ \\
\hline & $r$ odd & $\frac{q^{r-3}-1}{q+1}\left(q^{2 r-3}+q^{r-1}-q^{r-2}-1\right)$ \\
\hline $\mathbf{S}_{2 r}(q)$ & $r \geq 3$ & $\frac{q^{r-2}-1}{2}\left(q^{2(r-1)}-1\right)$ \\
\hline $\mathbf{O}_{7}(q)$ & $q \neq 2,3,4,9$ & $\frac{q-1}{\operatorname{gcd}(q-1,2)}\left(q^{4}-1\right)$ \\
\hline $\mathbf{O}_{2 r+1}(2)$ & $r \geq 5$ & $\frac{2\left(2^{r}-1\right)\left(2^{r-1}-1\right)}{6}\left(2^{2 r-2}-1\right)$ \\
\hline $\mathbf{O}_{2 r+1}(q)$ & $\begin{array}{l}r \geq 4 \\
q>2 \\
\end{array}$ & $\left(\frac{q^{2 r-4}-1}{q^{2}-1}-\frac{q^{r-2}-1}{q-1}\right)\left(q^{2 r-2}-1\right)$ \\
\hline $\boldsymbol{\Omega}_{8}^{+}(q)$ & $q \neq 2,4,9$ & $\frac{q-1}{\operatorname{gcd}(q-1,2)}\left(q^{5}+q^{3}-q^{2}-1\right)$ \\
\hline $\boldsymbol{\Omega}_{2 r}^{+}(2)$ & $r \geq 7$ & $\left(\frac{\left(2^{r-2}-1\right)\left(2^{r-3}-1\right)}{3}\left(2^{2 r-3}-2^{r-2}\right)\right.$ \\
\hline $\boldsymbol{\Omega}_{2 r}^{+}(q)$ & $\begin{array}{l}r \geq 5 \\
q>2\end{array}$ & $\left(\frac{q\left(q^{2 r-6}-1\right)}{q^{2}-1}-\frac{q^{r-3}-1}{q-1}-7\right)\left(q^{2 r-3}+q^{r-1}-q^{r-2}-1\right)$ \\
\hline $\mathbf{\Omega}_{8}^{-}(q)$ & $q \geq 4$ & $\frac{q^{2}-1}{\operatorname{gcd}\left(q^{2}-1,2\right)}\left(q^{5}-q^{3}+q^{2}-1\right)$ \\
\hline $\boldsymbol{\Omega}_{10}^{-}(q)$ & $q \geq 4$ & $\frac{q^{4}-1}{q+1}\left(q^{7}-q^{4}+q^{3}-1\right)$ \\
\hline $\boldsymbol{\Omega}_{2 r}^{-}(q)$ & $r \geq 6$ & $\left(\frac{q\left(q^{2 r-6}-1\right)}{q^{2}-1}-q^{r-3}-r+4\right)\left(q^{2 r-3}-q^{r-1}+q^{r-2}-1\right)$ \\
\hline
\end{tabular}

Table 4: Generic lower bound $b_{2}$ for $Q$-linear-large modules

(vii) $H=\mathbf{O}_{7}(q), q=2,3,4,9$; (viii) $H=\mathbf{O}_{n}(q), 9 \leq n \leq 11, q=2,3$;

(ix) $H=\boldsymbol{\Omega}_{8}^{+}(q), q=2,4,9$; (x) $H=\boldsymbol{\Omega}_{8}^{-}(q), q=2,3$; (xi) $H=\boldsymbol{\Omega}_{12}^{+}(2)$.

Lemma 5.5. Let $H$ and $W$ satisfy the $Q$-linear large hypothesis. Assume moreover that 
$O^{p}\left(L_{\chi}^{\infty}\right)$ is not one of the exceptions in Table [3, that is $H$ is not one of the groups in Remark 5.4. Let $b_{2}$ be as in Table 4 and $B$ as in Table 3 . Then either $2^{\left\lceil\left(b_{2}-1\right) / 2\right\rceil}>B^{3}$ or $H=\mathbf{O}_{7}(2)$ or $H=\mathbf{O}_{7}(3)$.

Proof. Suppose that $H \notin\left\{\mathbf{O}_{7}(2), \mathbf{O}_{7}(3)\right\}$. Taking $\log _{2}$ of both sides of the desired inequality, we see that it suffices to show $b_{2}>6 \log _{2} B+1$. Then it is a direct check that $b_{2}$ exceeds $6 \log _{2} B+1$ in each of the remaining cases.

Lemma 5.6. Let $H$ and $W$ satisfy the $Q$-linear large hypothesis and assume moreover that $H \notin\left\{\mathbf{L}_{4}(4), \mathbf{L}_{5}(2), \mathbf{L}_{6}(2), \mathbf{O}_{7}(2), \mathbf{O}_{9}(2), \boldsymbol{\Omega}_{8}^{ \pm}(2)\right\}$. Let $V$ be an irreducible $k H$-module. Then $2^{\lceil(\operatorname{dim} W-1) / 2\rceil}>(\operatorname{dim} V)^{3}$.

Proof. If $O^{p}\left(L_{\chi}^{\infty}\right)$ is not one of the exceptions in Table 3, that is, if $H$ is not one of the groups listed in Remark 5.4 and if $H \notin\left\{\mathbf{O}_{7}(2), \mathbf{O}_{7}(3)\right\}$, the result follows from Lemmas 5.5 and 5.2 . So we must consider each of the exceptions in turn.

We first simply apply the bound $\operatorname{dim} W \geq b_{1}(H)$ and find that $2^{\lceil(\operatorname{dim} W-1) / 2\rceil}>B^{3}$, where $B$ is the upper bound for the dimension of an irreducible $k H$-module unless $H$ is one of the following groups: $\mathbf{L}_{4}(4), \mathbf{L}_{5}(2), \mathbf{L}_{6}(2), \mathbf{U}_{7}(2), \mathbf{U}_{8}(2), \mathbf{O}_{7}(2), \mathbf{O}_{7}(3), \boldsymbol{\Omega}_{8}^{ \pm}(2), \mathbf{O}_{9}(2), \boldsymbol{\Omega}_{10}^{ \pm}(2)$, and $\mathbf{O}_{11}(2)$. We now apply a better lower bound, as in the proof of Lemma 5.5] using the $Q$-linear large hypothesis, to see that $\operatorname{dim} W \geq b_{1}\left(O^{p}\left(L_{\chi}^{\infty}\right)\right)\left[L: L_{\chi}\right]$, choosing of course a nonsolvable $O^{p}\left(L_{\chi}^{\infty}\right)$ and again find that $2^{\lceil(\operatorname{dim} W-1) / 2\rceil}>B^{3}$ unless $H$ is one of the groups listed as exceptions in the result.

Corollary 5.7. Let $H$ and $W$ satisfy the $Q$-linear large hypothesis and assume moreover that $H$ fixes a nondegenerate from on $W$; so if $G \subset \operatorname{Isom}(W)$ is the smallest classical group containing the image of $H, G$ is of type $B_{\ell}, C_{\ell}$ or $D_{\ell}$. Moreover, assume that $H \neq \mathbf{O}_{7}(2)$. Let $V_{G}(\lambda)$ be an irreducible $k G$-module with restricted highest weight $\lambda$, on which $H$ acts irreducibly. Then $r_{\lambda}=0$.

Proof. Suppose the contrary, that is, $r_{\lambda} \neq 0$. Note that Lemma 5.1 implies that $\ell \geq 3$.

Then by Lemma 4.9, $\operatorname{dim} V_{G}(\lambda) \geq 2^{\ell-1}$. Since $\ell=\left\lceil\frac{\operatorname{dim} W-1}{2}\right\rceil$, we have $\operatorname{dim} V_{G}(\lambda) \geq$ $2^{\left\lceil\frac{\operatorname{dim} W-1}{2}\right\rceil-1}$. Assume for the moment that $H \notin\left\{\mathbf{L}_{4}(4), \mathbf{L}_{5}(2), \mathbf{L}_{6}(2), \mathbf{O}_{9}(2), \boldsymbol{\Omega}_{8}^{ \pm}(2)\right\}$. Then by Lemma 5.6 , we have

$$
2^{\left\lceil\frac{\operatorname{dim} W-1}{2}\right\rceil-1}=(1 / 2) 2^{\left\lceil\frac{\operatorname{dim} W-1}{2}\right\rceil}>(1 / 2)\left(\operatorname{dim} V_{G}(\lambda)\right)^{3}>\operatorname{dim} V_{G}(\lambda),
$$

providing a contradiction.

Consider now the six groups omitted above. We argue as in the proof of Proposition 5.3 to obtain a lower bound for $\operatorname{dim} W$ and hence a lower bound for $\ell$. Moreover, we use a tighter upper bound for the dimension of a nontrivial irreducible $k H$-module, as indicated in Table 5 in the following section. In each of the remaining cases, $2^{\ell-1}>\operatorname{dim} V_{G}(\lambda)$, providing the required contradiction.

\section{The restricted case}

In this section, we establish the main theorem under the additional hypothesis that $V=V_{G}(\lambda)$ has a restricted highest weight $\lambda$. Recall that $V$ is a nontrivial irreducible $k H$-module. Initially, we will not require the hypothesis that $W$ is irreducible; indeed up through Lemma 6.5 we will assume only that $W$ is a $Q$-linear large $k H$-module. We will point out which point we assume the irreducibility hypothesis. Throughout this section let $e:=e(\lambda)$ and let $S$ be a nontrivial irreducible constituent of $\left.\operatorname{soc}\left(W_{\chi}\right)\right|_{L_{\chi}^{\infty}}$, for some $\chi \in Q^{*}, \chi \neq 1$, as in Definition 1.3

Proposition 6.1. Let $\ell$ be the rank of the classical group $C l(W)$. 
a) If $H \neq \mathbf{O}_{7}(2)$, then $e \leq(\ell+1) / 2$.

b) If $H \notin\left\{\mathbf{O}_{7}(2), \boldsymbol{\Omega}_{8}^{+}(2)\right\}$, then $e \leq 2$ or $e<\operatorname{dim} S$.

Proof. For (a), suppose the contrary: $e>\frac{\ell+1}{2}$. In case $C l(W)=\mathrm{SL}(W)$, Lemmas 5.1 and 5.2 imply that $\ell \geq 5$. Then Lemmas 3.2 and 4.8 show that $\operatorname{dim} V>2^{\ell} /(\ell+1) \geq 2^{\ell} / \ell^{2}$. In case $C l(W)$ is of type $B_{\ell}, C_{\ell}$ or $D_{\ell}$, then Lemma 4.9 gives $\operatorname{dim} V \geq 2^{\ell} / \ell^{2}$. Now $\ell \geq\left\lceil\frac{\operatorname{dim} W-1}{2}\right\rceil$ and $\ell \leq B$, so $\operatorname{dim} V \geq 2^{\left\lceil\frac{\operatorname{dim} W-1}{2}\right\rceil} / B^{2}$, which by Lemma 5.6 exceeds $B$, contradicting Lemma 5.2 , unless $H$ is one of $\mathbf{L}_{4}(4), \mathbf{L}_{5}(2), \mathbf{L}_{6}(2), \mathbf{O}_{9}(2)$, or $\boldsymbol{\Omega}_{8}^{ \pm}(2)$.

In the remaining cases, we consult the modular character tables in GAP08 and see that for $d$ the minimal dimension of an irreducible $k H$-module with $d \geq \operatorname{dim} W$, the value $2^{\lceil(d-1) / 2\rceil}$ exceeds the cube of the maximal-dimensional irreducible $k H$-module. This contradicts our inequality $\operatorname{dim} V \geq 2^{\ell} / \ell^{2}$, hence establishing the inequality.

For (b), we assume that $e \geq 3$. For the moment, assume in addition that $H$ is not one of the following groups (which are treated at the end of the proof):

(i) $\mathbf{L}_{4}(q), q=4,5,7,9$; (ii) $\mathbf{L}_{5}(q), q=2,4$; (iii) $\mathbf{L}_{6}(2)$;

(iv) $\mathbf{U}_{5}(q), q=4,5,7,8,9,11 ;$ (v) $\mathbf{U}_{6}(3)$; (vi) $\mathbf{U}_{7}(q), q=2,3$; (vii) $\mathbf{U}_{8}(q), q=2,3$;

(viii) $\mathbf{O}_{2 r+1}(q), r=4,5, q=2,3$; (ix) $\mathbf{O}_{7}(q), q=3,4,5,7,9,11,13$;

(x) $\boldsymbol{\Omega}_{2 r}^{ \pm}(q), r=5,6, q=2,3$; (xi) $\boldsymbol{\Omega}_{8}^{-}(q), q=2,3$; (xii) $\boldsymbol{\Omega}_{8}^{+}(q), q=3,4,5,7,8$.

(These groups are excluded from our first analysis for two reasons: either $O^{p}\left(L_{\chi}^{\infty}\right)$ provides an exception to the general polynomial bounds for $\operatorname{dim} W$ (see Table 3), or the polynomial bounds are too small because $q$ and $r$ are small.)

Now suppose $e \geq \operatorname{dim} S$. By (a), $e \leq(\ell+1) / 2$, and by Corollary 5.7, if $G=B_{\ell}, C_{\ell}$ or $D_{\ell}$, then $r_{\lambda}=0$. Then Proposition 4.7 and Lemma 4.9 imply that $\operatorname{dim} V \geq\left(\begin{array}{c}\ell+1 \\ e\end{array}\right)$ for $G=A_{\ell}$, and $\operatorname{dim} V \geq 2^{e}\left(\begin{array}{l}\ell \\ e\end{array}\right)$, for $G=B_{\ell}, C_{\ell}$ or $D_{\ell}$, the latter as long as $\ell \geq 7$. Applying Lemmas 5.1 and 5.2. we see that $\operatorname{dim} W>13$ and so $\ell \geq 7$.

Set $a=\left\lceil\left(\left[L: L_{\chi}\right]-1\right) / 2\right\rceil$ and $s=\operatorname{dim} S$, so that $e \geq s$. Since $\operatorname{dim} V \geq\left[L: L_{\chi}\right] \operatorname{dim} S$, we have $\ell \geq$ as and so

$$
\left(\begin{array}{l}
\ell \\
e
\end{array}\right) \geq\left(\begin{array}{l}
\ell \\
s
\end{array}\right) \geq\left(\begin{array}{c}
a s \\
s
\end{array}\right)
$$

Now if $s<a$, then $s^{2}<a s$ and by Lemma 3.1, we have $\left(\begin{array}{c}a s \\ s\end{array}\right) \geq(a s)^{s / 2}$. If on the other hand $s \geq a$ so that we have $(\ell+1) / 2 \geq e \geq s \geq a$, then $a^{2} \leq a s$ and we have

$$
\left(\begin{array}{l}
\ell \\
e
\end{array}\right) \geq\left(\begin{array}{c}
a s \\
s
\end{array}\right) \geq\left(\begin{array}{c}
a s \\
a
\end{array}\right) \geq(a s)^{a / 2} .
$$

Hence we now have

$$
(a s)^{m} \leq \operatorname{dim} V \leq B \text {, where } m=\min \{a / 2, s / 2\} \text { and } B \text { is as in Table } 3 \text {. }
$$

Taking $\log _{q}$ of this inequality gives

$$
m\left(\log _{q}(a s)\right) \leq \log _{q} B
$$

Now we refer to Table 2 for a lower bound for $a$, as well as the structure of $O^{p}\left(L_{\chi}^{\infty}\right)$. Now $s$ is greater than or equal to the Landazuri-Seitz-Zalesski, Guralnick-Tiep bound for the group $O^{p}\left(L_{\chi}^{\infty}\right)$ (see Table 3 denote this bound by $b_{1}\left(O^{p}\left(L_{\chi}^{\infty}\right)\right.$ ). Moreover, investigation of these lower bounds shows that $a \geq b_{1}\left(O^{p}\left(L_{\chi}^{\infty}\right)\right)$ in every case; hence we replace the inequality (11) by

$$
\frac{b_{1}\left(O^{p}\left(L_{\chi}^{\infty}\right)\right)}{2} \cdot \log _{q}\left(a \cdot b_{1}\left(O^{p}\left(L_{\chi}^{\infty}\right)\right)\right) \leq \log _{q} B
$$


Our assumption that $H$ is not one of the groups in (i) - (xii) above implies that there are no solutions to inequality (2).

We now consider the cases of (i) - (xii), where we argue that the exceptional multipliers do not generally appear in the group $O^{p}\left(L_{\chi}^{\infty}\right)$, and hence we can revert to the polynomial lower bound for the minimal dimension of a nontrivial irreducible $O^{p}\left(L_{\chi}^{\infty}\right)$ cross-characteristic representation. Moreover, we provide a more precise, improved upper bound for $\operatorname{dim} V$, deduced from [Sei90, Thm. 2.2].

By Lemmas 5.1] and 5.2, we see that $\ell \geq 7$. In particular, $e \leq \frac{\ell+1}{2} \leq \ell-3$ and so we may apply Proposition 4.7 to see that $\operatorname{dim} V \geq\left(\begin{array}{c}\ell+1 \\ e\end{array}\right)$, if $G=A_{\ell}$ and $\operatorname{dim} V \geq 2^{e}\left(\begin{array}{l}\ell \\ e\end{array}\right)$, if $G=B_{\ell}, C_{\ell}$ or $D_{\ell}$. Note as well that as $e \geq 3$, we have $2^{e}\left(\begin{array}{c}(c-1) / 2 \\ e\end{array}\right)<\left(\begin{array}{c}c-1 \\ e\end{array}\right)$ if $c$ is odd, and $2^{e}\left(\begin{array}{c}c / 2 \\ e\end{array}\right)<\left(\begin{array}{c}c-1 \\ e\end{array}\right)$ if $c$ is even. Hence we use in every case the lower bound for $\operatorname{dim} V$

$$
\operatorname{dim} V \geq 2^{e}\left(\begin{array}{c}
\left\lceil\frac{\operatorname{dim} W-1}{2}\right\rceil \\
e
\end{array}\right)
$$

and we take as our lower bound for $\operatorname{dim} W$, as in previous arguments, the product of $\left[L: L_{\chi}\right]$ and the minimal dimension of a nontrivial cross-characteristic representation of $O^{p}\left(L_{\chi}^{\infty}\right)$. For the upper bound, we do not use the approximations in Table 3, but rather the actual upper bounds given by [Sei90, Thm. 2.1]. We record these values for each of the groups in Table 5.

\begin{tabular}{|l|l|}
\hline$H$ & upper bound for $\operatorname{dim} V$ \\
\hline $\mathbf{L}_{r+1}(q)$ & $\frac{\left(q^{r+1}-1\right)\left(q^{r}-1\right) \cdots\left(q^{2}-1\right)}{(q-1)^{r}}$ \\
\hline $\mathbf{U}_{r+1}(q), r$ odd & $\frac{\left(q^{r+1}-(-1)^{r+1}\right)\left(q^{r}-(-1)^{r}\right) \cdots\left(q^{2}-1\right)}{\left(q^{2}-1\right)^{(r+1) / 2}} \cdot \frac{q^{2}-1}{q^{2}-q+1}$ \\
$\mathbf{U}_{r+1}(q), r$ even & $\frac{\left(q^{r+1}-(-1)^{r+1}\right)\left(q^{r}-(-1)^{r}\right) \cdots\left(q^{2}-1\right)}{(q+1)\left(q^{2}-1\right)^{r / 2}} \cdot \frac{q^{2}-1}{q^{2}-q+1}$ \\
\hline $\mathbf{O}_{2 r+1}(q)$ & $\frac{\left(q^{2 r}-1\right)\left(q^{2 r-2}-1\right) \cdots\left(q^{2}-1\right)}{(q-1)^{r}}$ \\
\hline $\mathbf{\Omega}_{2 r}^{+}(q)$ & $\frac{\left(q^{r}-1\right)\left(q^{2 r-2}-1\right)\left(q^{2 r-4}-1\right) \cdots\left(q^{2}-1\right)}{(q-1)^{r}}$ \\
\hline $\mathbf{\Omega}_{2 r}^{-}(q)$ & $\frac{\left(q^{r}+1\right)\left(q^{2 r-2}-1\right)\left(q^{2 r-4}-1\right) \cdots\left(q^{2}-1\right)}{(q+1)(q-1)^{r-1}}$ \\
\hline
\end{tabular}

Table 5: Upper bound for $\operatorname{dim} V$

For $H=\mathbf{L}_{4}(q), q=4,5,7,9$, we first note that $O^{p}\left(L_{\chi}^{\infty}\right) \supset \mathrm{SL}_{2}(q)$, whose minimal dimensional nontrivial representation over $k$ has dimension $q-1$ if $q$ is even, and $(q-1) / 2$ if $q$ is odd. So we have

$$
\frac{\left(q^{4}-1\right)\left(q^{3}-1\right)\left(q^{2}-1\right)}{(q-1)^{3}} \geq \operatorname{dim} V \geq 2^{e}\left(\begin{array}{c}
\frac{\left(q^{3}-1\right)(q-1)-\operatorname{gcd}(2, q-1)}{2 \operatorname{gcd}(2, q-1)} \\
e
\end{array}\right) .
$$

One now checks that for $e \geq 3$, and $q=4,5,7,9$, the above inequality is never satisfied. 
For $H=\mathbf{L}_{5}(4)$, the minimal dimensional module for $O^{p}\left(L_{\chi}^{\infty}\right)$ is of dimension at least 4 . Hence, $\operatorname{dim} W \geq\left(4^{4}-1\right) \cdot 4$ and one checks that $2^{e}\left(\begin{array}{c}509 \\ e\end{array}\right)$ exceeds $\left(q^{5}-1\right)\left(q^{4}-1\right)\left(q^{3}-1\right)\left(q^{2}-\right.$ $1) /(q-1)^{4}$, when $q=4$ and $e \geq 3$, contradicting the inequality (3). For $H=\mathbf{L}_{6}(2)$, a similar analysis gives a contradiction. For the group $H=\mathbf{L}_{5}(2)$ a slightly finer analysis is required. Here we see that $O^{p}\left(L_{\chi}^{\infty}\right) \supset \mathrm{SL}_{3}(2)$ and the minimal dimension of a cross-characteristic representation of this group is 3 . Hence $\operatorname{dim} W \geq 3\left(2^{4}-1\right)$, while consulting the modular character tables in GAP08, we see that the maximal possible dimension for $V$ is 1240 . These values contradict the inequality (3).

Turn now to the unitary groups. For $\mathbf{U}_{5}(q)$, where we must consider the cases $4 \leq$ $q \leq 11$, we have $\operatorname{dim} W \geq\left(q^{5}+q^{2}\right) \cdot b_{1}\left(\mathbf{U}_{2}(q)\right)=\left(q^{5}+q^{2}\right) \cdot \frac{q-1}{\operatorname{gcd}(2, q-1)}$. (As above, we have that $O^{p}\left(L_{\chi}^{\infty}\right) \supset \mathrm{SL}_{2}(q)$ in all cases and so this is indeed an accurate lower bound.) A direct calculation shows that $2^{e}\left({ }^{\lceil(\operatorname{dim} W-1) / 2\rceil}\right)$ exceeds the upper bound for $\operatorname{dim} V$ as given in Table 5. For $H=\mathbf{U}_{6}(3)$, we may use the lower bound of Table $4, \operatorname{dim} W \geq b_{2} \geq$ $\left(3^{7}+3^{4}-3^{3}-1\right)$. Again, a direct check gives a contradiction to the inequality (3) and the upper bound of Table 5 .

For $H=\mathbf{U}_{7}(q), q=2,3$, since $\mathbf{U}_{3}(2)$ is solvable, we have $\operatorname{dim} W \geq\left(2^{9}+2^{4}\right) b_{1}\left(\mathbf{U}_{4}(2)\right)=$ $4\left(2^{9}+2^{4}\right)$, when $q=2$ and $\operatorname{dim} W \geq\left(3^{9}-3^{5}+3^{4}-1\right) b_{1}\left(\mathbf{U}_{3}(3)\right)=6\left(3^{9}-3^{5}+3^{4}-1\right)$, when $q=3$. In each case, we obtain a contradiction by comparing the upper and lower bounds for $\operatorname{dim} V$. A similar analysis for the groups $H=\mathbf{U}_{8}(q), q=2,3$, yields a contradiction as well.

For $\mathbf{O}_{7}(q)$, with $q=3,4,5,7,8,9,11,13$, we have $\operatorname{dim} V \leq \frac{\left(q^{6}-1\right)\left(q^{4}-1\right)\left(q^{2}-1\right)}{(q-1)^{3}}$, while

$$
\operatorname{dim} W \geq \min \left\{\left(q^{4}-1\right) b_{1}\left(\mathbf{O}_{3}(q)\right),\left(q^{4}-q^{2}\right) b_{1}\left(\mathbf{\Omega}_{4}^{-}(q)\right),\left(q^{4}+q^{2}\right) b_{1}\left(\mathbf{\Omega}_{4}^{+}(q)\right)\right\} .
$$

Hence $\operatorname{dim} W \geq \min \left\{\left(q^{4}-1\right) \frac{q-1}{\operatorname{gcd}(q-1,2)},\left(q^{4}-q^{2}\right) \frac{q^{2}-1}{\operatorname{gcd}(q-1,2)}\right\}$. Note that we use the second of these terms in the case $q=3$, since $\mathbf{O}_{3}(3)$ is solvable. Now one checks that the lower bound given by (3) exceeds the upper bound in every case.

For $H=\Omega_{8}^{+}(q)$, with $q=3,4,5,7,8$, we have $\operatorname{dim} V \leq \frac{\left(q^{4}-1\right)\left(q^{6}-1\right)\left(q^{4}-1\right)\left(q^{2}-1\right)}{(q-1)^{4}}$, while $\operatorname{dim} W \geq \min \left\{\left(q^{5}+q^{3}-q^{2}-1\right) b_{1}\left(\boldsymbol{\Omega}_{4}^{+}(q)\right),\left(q^{5}-q^{2}\right) b_{1}\left(\mathbf{O}_{5}(q)\right)\right\}$. Note that we use the latter expression if $q=3$, since $\boldsymbol{\Omega}_{4}^{+}(3)$ is solvable. Now $b_{1}\left(\boldsymbol{\Omega}_{4}^{+}(q)\right)=\frac{q-1}{\operatorname{gcd}(q-1,2)}$ and $b_{1}\left(\mathbf{O}_{5}(q)\right)=\frac{q^{2}-1}{2}$ if $q$ is odd, respectively, $\frac{q\left(q^{2}-1\right)(q-1)}{2(q+1)}$ for $q$ even. In particular, $\operatorname{dim} W \geq\left(q^{5}+q^{3}-q^{2}-1\right)(q-$ $1) / \operatorname{gcd}(q-1,2)$ in all cases. Now one checks that $2^{e}\left(\begin{array}{c}\lceil(\operatorname{dim} W-1) / 2\rceil \\ e\end{array}\right)$ exceeds $\operatorname{dim} V$ for all $e \geq 3$.

For $H=\mathbf{O}_{9}(q), q=2,3$, we have

$\operatorname{dim} W \geq \min \left\{\left(q^{6}-1\right) b_{1}\left(\mathbf{O}_{5}(q)\right),\left(q^{6}+q^{3}\right) /\left(1+\delta_{2, p}\right) b_{1}\left(\boldsymbol{\Omega}_{6}^{+}(q)\right),\left(q^{6}-q^{3}\right) /\left(1+\delta_{2, p}\right) b_{1}\left(\mathbf{\Omega}_{6}^{-}(q)\right)\right\}$.

Recall that $b_{1}\left(\boldsymbol{\Omega}_{6}^{+}(q)\right)=b_{1}\left(\mathbf{L}_{4}(q)\right)$ and $b_{1}\left(\boldsymbol{\Omega}_{6}^{-}(q)\right)=b_{1}\left(\mathbf{U}_{4}(q)\right)$. Note that $O^{p}\left(L_{\chi}^{\infty}\right)$ contains a subgroup isomorphic to either the orthogonal group $\mathrm{O}_{5}(2)$, or the linear group $\mathrm{SL}_{4}(2)$, or the unitary group $\mathrm{SU}_{4}(2)$. We now consult the modular character tables in GAP08 to find that the minimal dimensional representations of these groups are 7,7 and 5 , respectively, and hence $\operatorname{dim} W \geq \min \left\{7\left(2^{6}-1\right), 7(1 / 2)\left(2^{6}+2^{3}\right), 5(1 / 2)\left(2^{6}-2^{3}\right)\right\}$, while (again using [GAP08]) the maximal dimension of a nontrivial cross-characteristic representation of $\mathbf{O}_{9}(2)$ is 68850 . This contradicts the inequality (3). For $q=3$, we use an analogous argument.

For $H=\Omega_{10}^{ \pm}(q)$, with $q=2,3$, we have

$$
\operatorname{dim} W \geq \min \left\{\left(q^{7} \pm q^{4} \mp q^{3}-1\right) b_{1}\left(\boldsymbol{\Omega}_{6}^{ \pm}(q)\right),\left(q^{7} \mp q^{3}\right) b_{1}\left(\mathbf{O}_{7}(q)\right)\right\}
$$

Now we use the fact that $O^{p}\left(L_{\chi}^{\infty}\right) \supset \mathrm{SL}_{4}(q)$, respectively $\mathrm{SU}_{4}(q)$, when $H$ is of + , respectively - type. We can then consult the modular character tables in GAP08 to obtain the precise lower bounds for the dimensions of the irreducible representations of these groups. We have $b_{1}\left(\mathrm{SL}_{4}(2)\right)=28, b_{1}\left(\mathrm{SU}_{4}(2)\right)=34$, and $b_{1}\left(\mathrm{SU}_{4}(3)\right)=15$. Hence $\operatorname{dim} W \geq\left(q^{7} \pm q^{4} \mp q^{3}-\right.$ 
1) $b_{1}\left(\boldsymbol{\Omega}_{6}^{ \pm}(q)\right)$. We use the upper bound $\operatorname{dim} V \leq 68850$, which is found in the character table for $H$. Now one can check that in each case inequality (3) fails.

For $H=\mathbf{O}_{11}(q), q=2,3$, we have $\operatorname{dim} V \leq \frac{\left(q^{10}-1\right)\left(q^{8}-1\right)\left(q^{6}-1\right)\left(q^{4}-1\right)\left(q^{2}-1\right)}{(q-1)^{5}}$, while $\operatorname{dim} W \geq$ $\min \left\{\left(q^{8} \pm q^{4}\right) b_{1}\left(\boldsymbol{\Omega}_{8}^{ \pm}(q)\right) /\left(1+\delta_{2, q}\right),\left(q^{8}-1\right) b_{1}\left(\mathbf{O}_{7}(q)\right)\right\}$. Using that $O^{p}\left(L_{\chi}^{\infty}\right)$ contains the almost simple group $\boldsymbol{\Omega}_{8}^{+}(2)$, and not a double cover, when $p=2$, we see that we may use the lower bound $b_{1}\left(\boldsymbol{\Omega}_{8}^{+}(2)\right)=28$. (See the modular character tables in GAP08.) It is then a direct check to see that in each case the inequality (3) fails.

Finally, for $H=\Omega_{12}^{ \pm}(q), q=2,3$, we have $\operatorname{dim} V \leq \frac{\left(q^{6} \mp 1\right)\left(q^{10}-1\right)\left(q^{8}-1\right)\left(q^{6}-1\right)\left(q^{4}-1\right)\left(q^{2}-1\right)}{(q \mp 1)(q-1)^{5}}$. Here $\left.\operatorname{dim} W \geq \min \left\{\left(q^{9} \mp q^{4}\right) b_{1}\left(\boldsymbol{\Omega}_{9}(q)\right),\left(q^{9} \pm q^{5} \mp q^{4}-1\right)\right) b_{1}\left(\boldsymbol{\Omega}_{8}^{ \pm}(q)\right)\right\}$. Again, one can work with the almost simple group $\boldsymbol{\Omega}_{8}^{+}(2)$, and in the other cases use the general polynomial bound for $b_{1}\left(O^{p}\left(L_{\chi}^{\infty}\right)\right)$. In every case, inequality (3) fails.

Remark. It is perhaps worth noting that the analysis of the previous proof does not require that $S$ be an $L_{\chi}^{\infty}$-submodule of $W_{\chi}$, but rather simply that we have a nonlinear irreducible composition factor. This observation could be useful when one considers the case when $W$ is not a $Q$-linear large $k H$-module.

Lemma 6.2. Assume that $\operatorname{dim} S>e$ and that $e \leq(\ell+1) / 2$. Then there exists a $P_{\chi}$-invariant submodule of $V$ of dimension at most $(\operatorname{dim} S)^{e}$. In particular, $\operatorname{dim} V \leq(\operatorname{dim} S)^{e}\left[H: P_{\chi}\right]$.

Proof. By Proposition 6.1, we have that $e \leq(\ell+1) / 2$ and $e<\operatorname{dim} S$ and Corollary 5.7 assures that either $r_{\lambda}=0$ or $G$ is of type $A_{\ell}$. Hence, if $G$ is of type $A_{\ell}$, we have $l=$ $\sum_{i=1}^{\operatorname{dim} S-1} a_{i} l_{i}+\sum_{i=\ell-\operatorname{dim} S+2}^{\ell} a_{i} l_{i}$ and $\lambda=\sum_{i=1}^{\operatorname{dim} S-1} a_{i} l_{i}$, otherwise.

We first consider the case where $S$ is a totally singular subspace of $W$. Then $\operatorname{Stab}_{G}(S)$ is a parabolic subgroup $P_{G}=Q_{G} L_{G}$, with $\left[L_{G}, L_{G}\right] \cong \mathrm{GL}(S) \times \operatorname{Isom}\left(S^{\perp} / S\right)$. If $r_{\lambda} l_{\lambda}=0$, then without loss of generality, we take the GL $(S)$ factor to be the subsystem subgroup of $G$ corresponding to the set of simple roots $\left\{\alpha_{1}, \ldots, \alpha_{\operatorname{dim} S-1}\right\}$ (or $\left\{\alpha_{\ell}, \ldots, \alpha_{\ell-\operatorname{dim} S+2}\right\}$ if $r_{\lambda} \neq 0$ ). In this case, $C_{V}\left(Q_{G}\right)$ is a $P_{G}$-invariant submodule on which, by $\underline{\text { Smi82 }}, L_{G}$ acts irreducibly with highest weight $\left.\lambda\right|_{L_{G}}$. Moreover, $P_{\chi} \leq \operatorname{Stab}_{G}(S)=P_{G}$ and so $P_{\chi}$ also acts on $C_{V}\left(Q_{G}\right)$. The irreducibility of $\left.V\right|_{H}$ then implies that $\operatorname{dim} V \leq \operatorname{dim} C_{V}\left(Q_{G}\right)\left[H: P_{\chi}\right]$. By Lemma 4.7 $\operatorname{dim} C_{V}\left(Q_{G}\right) \leq(\operatorname{dim} S)^{e}$, and the result follows. In the case where $S$ is totally singular and $r_{\lambda} l_{\lambda} \neq 0$ (so $G$ is of type $A_{\ell}$ ), write $\lambda=\lambda_{\ell}+\lambda_{r}$, where $\lambda_{\ell}$ has support on $\left\{\alpha_{1}, \ldots, \alpha_{l_{\lambda}}\right\}$ and $\lambda_{r}$ has support on $\left\{\alpha_{\ell-r_{\lambda}+1}, \ldots, \alpha_{\ell}\right\}$. In particular, $e(\lambda)=e\left(\lambda_{\ell}\right)+e\left(\lambda_{r}\right)<\operatorname{dim} S$ and we replace the GL $(S)$ factor in the above argument by the subsystem subgroup of $G$ corresponding to the set of simple roots $\left\{\alpha_{1}, \ldots, \alpha_{e\left(\lambda_{l}\right)}, \alpha_{\ell}, \ldots, \alpha_{\ell-\operatorname{dim} S-e\left(\lambda_{l}\right)-1}\right\}$. Now apply Lemma4.7 once again to obtain the result.

We now consider the case where $S$ is not totally singular. In particular, $G$ preserves a nondegenerate symplectic or quadratic form, so $r_{\lambda}=0$, and the existence of the nontrivial $Q$-character $\chi$ implies that $p=2$, and so $\operatorname{char}(k) \neq 2$. Hence, the irreducibility of $\left.S\right|_{L_{\chi}}$ implies that $S$ is a nondegenerate subspace with respect to the bilinear form on $W$. In this case, we have $P_{\chi}<\left(\operatorname{Isom}(S) \times \operatorname{Isom}\left(S^{\perp}\right)\right) \cap G$. As in the previous case, we may assume without loss of generality that the restriction of $l$ to a maximal torus of $\operatorname{Isom}\left(S^{\perp}\right)$ is the zero weight. Moreover, taking a standard embedding of $\left(\operatorname{Isom}(S) \times \operatorname{Isom}\left(S^{\perp}\right)\right) \cap G$ in $G$, we have that the maximal vector $v^{+}$of $V$ with respect to a fixed Borel subgroup of $G$ is a maximal vector for a Borel subgroup of $\operatorname{Isom}(S)$ with the highest weight given by restricting $l$ to the standard torus of $\operatorname{Isom}(S)$. We have the $\left(\left(\operatorname{Isom}(S) \times \operatorname{Isom}\left(S^{\perp}\right)\right) \cap G\right)$-invariant submodule $Y:=\left(\left(\operatorname{Isom}(S) \times \operatorname{Isom}\left(S^{\perp}\right)\right) \cap G\right) \cdot v^{+}$, which is an image of the Weyl module for $\operatorname{Isom}(S)$ of highest weight $l$. Hence, as in the proof of Lemma 4.7 $\operatorname{dim} Y \leq(\operatorname{dim} S)^{e}$.

Proposition 6.3. Assume that $H \notin\left\{\mathbf{O}_{7}(2), \mathbf{O}_{9}(2), \boldsymbol{\Omega}_{8}^{+}(2), \mathbf{L}_{5}(2), \mathbf{L}_{6}(2)\right\}$. Then $e=1$ or $e=2$.

Proof. We assume $e \geq 3$ and arrive at a contradiction. By Proposition 6.1, we have $\operatorname{dim} S>e$ and $e \leq(\ell+1) / 2$, and hence we may apply Lemma 6.2. 
We continue with the notation as established above. In particular, we have $\operatorname{dim} W \geq[L$ : $\left.L_{\chi}\right] \operatorname{dim} S$; hence we have $\operatorname{dim} W>13$ and so $\ell \geq 7$.

Now note that for all natural numbers $s, a, e \geq 1$, with $e \leq a$, we have

$$
\left(\begin{array}{c}
s a \\
e
\end{array}\right)=\frac{(s a)(s a-1) \cdots(s a-e+1)}{e !}=s^{e} \frac{a(a-1 / s) \cdots(a-(e-1) / s)}{e !} \geq s^{e}\left(\begin{array}{l}
a \\
e
\end{array}\right) .
$$

Set $s=\operatorname{dim} S$ and $a=\left\lceil\left(\left[L: L_{\chi}\right]-1\right) / 2\right\rceil$ if $G$ is of type $B_{\ell}, C_{\ell}$ or $D_{\ell}$ and let $a=\left[L: L_{\chi}\right]-1$ if $G$ is of type $A_{\ell}$. As $\ell=\lceil(\operatorname{dim} W-1) / 2\rceil$, or $\ell=\operatorname{dim} W-1$, respectively, we have $\ell \geq s a$.

By Lemma 4.7 Proposition 6.1, Lemma 6.2 and the above remarks, we have

$$
(\operatorname{dim} S)^{e}\left(\begin{array}{c}
a \\
e
\end{array}\right) \leq\left(\begin{array}{c}
s a \\
e
\end{array}\right) \leq\left(\begin{array}{l}
\ell \\
e
\end{array}\right) \leq \operatorname{dim} V \leq(\operatorname{dim} S)^{e}\left[H: P_{\chi}\right],
$$

if $G$ is of type $A_{\ell}$, and

$$
(\operatorname{dim} S)^{e} 2^{e}\left(\begin{array}{l}
a \\
e
\end{array}\right) \leq 2^{e}\left(\begin{array}{c}
a s \\
e
\end{array}\right) \leq 2^{e}\left(\begin{array}{l}
\ell \\
e
\end{array}\right) \leq \operatorname{dim} V \leq(\operatorname{dim} S)^{e}\left[H: P_{\chi}\right],
$$

otherwise. So we have

$$
\left(\begin{array}{c}
{\left[L: L_{\chi}\right]-1} \\
e
\end{array}\right) \leq\left[H: P_{\chi}\right]
$$

or

$$
2^{e}\left(\begin{array}{c}
\left.\left\lceil\left(L: L_{\chi}\right]-1\right) / 2\right\rceil \\
e
\end{array}\right) \leq\left[H: P_{\chi}\right]
$$

according to whether $G$ has type $A_{\ell}$ or not. Note that $\left[H: P_{\chi}\right]=[H: P]\left[L: L_{\chi}\right]$. Also, as $e \geq 3$ and $e \leq(\ell+1) / 2$, we have $\left(\begin{array}{l}\ell \\ e\end{array}\right) \geq\left(\begin{array}{l}\ell \\ 3\end{array}\right)$.

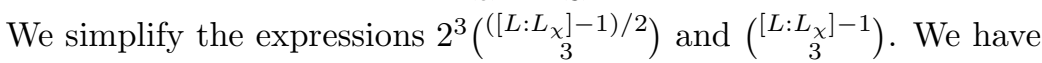

$$
\frac{1}{6}\left[L: L_{\chi}\right]\left(\left[L: L_{\chi}\right]-3\right)\left(\left[L: L_{\chi}\right]-6\right)<2^{3}\left(\begin{array}{c}
\left(\left[L: L_{\chi}\right]-1\right) / 2 \\
3
\end{array}\right) ;
$$

and

$$
\frac{1}{6}\left[L: L_{\chi}\right]\left(\left[L: L_{\chi}\right]-2\right)\left(\left[L: L_{\chi}\right]-4\right)<\left(\begin{array}{c}
{\left[L: L_{\chi}\right]-1} \\
3
\end{array}\right) .
$$

Hence we must now solve the inequalities

$$
\frac{1}{6}\left(\left[L: L_{\chi}\right]-3\right)\left(\left[L: L_{\chi}\right]-6\right) \leq[H: P]
$$

and

$$
\frac{1}{6}\left(\left[L: L_{\chi}\right]-2\right)\left(\left[L: L_{\chi}\right]-4\right) \leq[H: P] .
$$

The proposition follows from the next claim in case $e \leq a$; that is the claim shows that there are no solutions to the inequalities (6) and (7), and hence gives the desired contradiction.

Claim 6.4. For every $H$ as in the statement of the proposition, we have

$$
\left(\left[L: L_{\chi}\right]-3\right)\left(\left[L: L_{\chi}\right]-6\right)>6[H: P] .
$$

Proof of claim: Note that $[H: P]$ is the number of singular 1-spaces in $N$, the natural (projective) module for $H$ (and hence can be deduced from the last column of Table 2). Comparing the degrees of the polynomial expressions obtained by using the minimal possible value for 
$\left[L: L_{\chi}\right]$ and the actual value of $[H: P]$ gives one a clear idea that the claim is reasonable. It is a tedious but straightforward exercise to verify the inequality for each of the groups $H$ covered by the proposition. This completes the proof of the claim.

Now consider the case where $e>a$; that is, $e>\left[L: L_{\chi}\right]-1$, if $G$ is of type $A_{\ell}$ and $e>\left\lceil\left(\left[L: L_{\chi}\right]-1\right) / 2\right\rceil$, otherwise. Here we refer to the proof of Proposition 6.1 the argument given there applies and shows that under the condition that $e>a$, the general lower bound of $2^{e}\left(\begin{array}{l}\ell \\ e\end{array}\right)$ or $\left(\begin{array}{l}\ell \\ e\end{array}\right)$ exceeds the upper bound $B$ for $\operatorname{dim} V$.

This completes the proof of the proposition.

Lemma 6.5. Assume that $H$ is one of the groups $\mathbf{O}_{7}(2), \boldsymbol{\Omega}_{8}^{+}(2), \mathbf{O}_{9}(2), \mathbf{L}_{5}(2)$ or $\mathbf{L}_{6}(2)$, and that $W$ satisfies the $Q$-linear large hypothesis. Let $V$ be a restricted irreducible $k G$-module with highest weight $\lambda$, on which $H$ acts irreducibly. Then $e(\lambda) \leq 2$.

Proof. Set $e=e(\lambda)$. We assume that $e \geq 3$, and arrive at a contradiction in each case.

We first consider the group $H=\mathbf{O}_{7}(2)$. The $Q$-linear large hypothesis is satisfied only if $O^{p}\left(L_{\chi}^{\infty}\right)$ is isomorphic to $\boldsymbol{\Omega}_{4}^{-}(2)$, since in the other cases, $L_{\chi}$ is solvable. Here the orbit length $\left[L: L_{\chi}\right]$ is 6 . Moreover, as the group $H$ may have an exceptional Schur multiplier, we must allow for the possibility that $L_{\chi}^{\infty}$ is the double cover of the group Alt 5 . We also note that the maximal possible dimension for $V$ is 720 (see GAP08)). Since $8\left(\begin{array}{l}d \\ 3\end{array}\right)$ exceeds 720 for all $d \geq 21$, we see that $\operatorname{dim} W<21$. Consulting the modular character tables for $H$ in GAP08, we see that the only $k H$-module that is both $Q$-linear large and of dimension less than 21 is either of dimension 15 , when $\operatorname{char}(k) \neq 3$ or of dimension 14 when $\operatorname{char}(k)=3$; moreover, we have that $G=B_{7}$ when $\operatorname{char}(k) \neq 3$ and $G=D_{7}$, when $\operatorname{char}(k)=3$. We also note that the module $W$ in each case is a module for $\operatorname{Sp}_{6}(2)$ and not for the double cover. Hence, since we know that the weight $\lambda$ satisfies $r_{\lambda}=0$, the module $V$ is a module for the group $\mathrm{SO}(W)$ and so $V$ is also a representation of the group $\mathrm{Sp}_{6}(2)$, and not the double cover. Hence our upper bound now becomes 512 , rather than 720 . Now as $16\left(\begin{array}{l}7 \\ 4\end{array}\right)>512$, we see that $e=3$. We may refer to Lüb01 to see that the only module for $G=B_{7}$, when $\operatorname{char}(k) \neq 2,3$, with restricted highest weight $\lambda$ satisfying $e(\lambda)=3, r_{\lambda}=0$ and $\operatorname{dim} V \leq 512$ is the module with highest weight $\lambda_{3}$, of dimension 455 . Finally, we conclude by noting that there is no irreducible $k H$-module of dimension 455 .

In case $\operatorname{char}(k)=3$, the maximal dimension of an irreducible $k H$-module is 405 . Here $G=D_{7}$ and by [Lüb01] we see that the only $k G$-module when $\operatorname{char}(k)=3$, with restricted highest weight $\lambda$ satisfying $e(\lambda)=3, r_{\lambda}=0$ and $\operatorname{dim} V \leq 405$, is the module with highest weight $\lambda_{3}$, of dimension 364. But now we refer to GAP08 to see that there is no such 3modular irreducible representation of $H=\mathbf{O}_{7}(2)$. This completes the consideration of the case $H=\mathbf{O}_{7}(2)$.

Now consider the case $H=\boldsymbol{\Omega}_{8}^{+}(2)$; here $\operatorname{dim} V \leq 6075$. Here we must have $L_{\chi}^{\infty}=\mathbf{O}_{5}(2)$ and $\left[L: L_{\chi}\right]=28$. Since the 3 -modular representations of $\mathbf{O}_{5}(2)$ are of degrees $2,3,4,6$ and 9 , we see that $\operatorname{dim} W \geq 2 \cdot 28$. But comparing this with the 3 -modular character table of $H$, we conclude that $\operatorname{dim} W \geq 104$. But then $8\left({ }^{[(\operatorname{dim} W-1) / 2\rceil}\right)$ exceeds 6075 .

Hence we are in the situation where $\operatorname{char}(k) \neq 2,3$. Here the character degrees of $\mathbf{O}_{5}(2)$ are $4,5,8,9$, and 10. So we have $\operatorname{dim} W \geq 4 \cdot 28=112$. But then $8(\lceil(\operatorname{dim} W-1) / 2\rceil)$ exceeds 6075 .

For the group $\mathbf{O}_{9}(2)$, the minimal degree of a $Q$-linear large representation is 118 and so $\ell \geq 59$. Also Propositions 6.1 and 4.7 give a lower bound for $\operatorname{dim} V$ which exceeds the maximal degree of a cross-characteristic irreducible representation, which is 68850 .

For the group $\mathbf{L}_{5}(2)$, a similar argument applies, given that the minimal dimension of a $Q$-linear large representation is 94 and the maximal dimension is 1240 .

Finally, for the group $\mathbf{L}_{6}(2)$, the relevant lower, respectively upper, bounds are 217 , resp. 41664 again leading to a contradiction. This completes the proof of the lemma. 
All of the precise upper and lower bounds used above are obtained from GAP08, if not mentioned otherwise.

Combining Proposition 6.3 and Lemma 6.5. we now see that if $V$ is a restricted irreducible $k G$-module with highest weight $\lambda$ on which $H$ acts irreducibly, then $e(\lambda) \leq 2$. Our aim now is to see that in fact $e(\lambda)=1$, that is, $V$ is simply the module $W$ or its dual $W^{*}$. This result plays a key role when we consider the more general case of nonrestricted weights in the next section. The proof is based upon results of [MT01] and [MMT02, which treat the irreducibility of tensor squares, and the symmetric and alternating square of irreducible $k H$-modules. Thus, for the next two results we assume that $W$ is a nontrivial irreducible $k H$-module.

Lemma 6.6. Let $W$ be a nontrivial irreducible $k H$-module and assume $W$ and $H$ satisfy the $Q$-linear large hypothesis. Let $V$ be an irreducible $k G$-module with restricted highest weight $\lambda$ satisfying $e(\lambda)=2$. Then either $\left.V\right|_{H}$ is reducible or $\operatorname{char}(k)=3, H=\mathbf{O}_{2 r+1}(2), r \geq 4$ or $H=\Omega_{2 r}^{ \pm}(2), r \geq 5$. Moreover, $W$ is equipped with an $H$-invariant bilinear or quadratic form, and $V$ is the maximal-dimensional composition factor of $\Lambda^{2}(W)$ or $S^{2}(W)$.

Proof. Our hypothesis implies that $V$ is either the heart (i.e., the maximal-dimensional $k G$ composition factor) of the symmetric- or alternating square of $W$, or that $V$ is the heart of $W \otimes W^{*}$. The content of Theorems 1.2 and 3.1 of [MMT02 is that $\left.V\right|_{H}$ is reducible unless one of the following holds:

(i) $W$ is a Weil module for $H$ of type $\mathbf{S}$ or $\mathbf{U}$, or

(ii) $H$ is "small" (precisely given in MM98, Table 3.1] or [MMT02, Table 3.2]), or

(iii) $H$ is not of type $\mathbf{L}$ and $q \in\{2,4,8\}$.

In the last case, a precise bound for $\operatorname{dim} V$ is given. The Weil modules for the symplectic and unitary groups do not satisfy the $Q$-linear large hypothesis; nor do the small linear group examples, listed in [MM98, Table 3.1]. Thus [MMT02, 1.2, 5.3, 5.5, 5.7, 5.8, 5.13,] imply that $\left.V\right|_{H}$ is reducible unless possibly $V$ is the heart of the symmetric or alternating square of $W$, $G$ is of type $B_{\ell}, C_{\ell}$ or $D_{\ell}$, and moreover one of following is true:

a) $H=\mathbf{U}_{r+1}(2)$, and $\operatorname{dim} V \leq 2^{r+1}\left(2^{r+1}-(-1)^{r+1}\right)\left(2^{r}+(-1)^{r}\right)\left(2^{r-2}+1\right) / 3$.

b) $H=\mathbf{O}_{2 r+1}(q)$ with $q \in\{4,8\}$, or $q=2, r \geq 4$ and $\operatorname{char}(k) \neq 3$; moreover $\operatorname{dim} W \leq$ $2\left(q^{2 r-1}-1\right)$.

c) $H=\mathbf{O}_{2 r+1}(2), r \geq 4$, and $\operatorname{char}(k)=3$; moreover $\operatorname{dim} V \leq 2^{4 r-8}\left(2^{2 r}-1\right)\left(2^{2 r-2}-1\right) / 5$.

d) $H=\Omega_{2 r}^{ \pm}(q)$ with $q \in\{2,4\}$ and $\operatorname{char}(k) \neq 3$ if $(r, q)=(4,2)$; moreover $\operatorname{dim} V \leq$ $\frac{1}{2} q^{2 r-2}\left(q^{r} \mp 1\right)\left(q^{r-1} \pm 1\right) /(q-1)$.

e) $H=\Omega_{2 r}^{ \pm}(2), r \geq 5$, and $\operatorname{char}(k)=3$; moreover $\operatorname{dim} V \leq 2^{4 r-6}\left(2^{2} \mp 1\right)\left(2^{2 r-2}-1\right)\left(2^{r-2} \mp\right.$ 1) $/ 15$.

f) $H=\mathbf{O}_{7}(2), \operatorname{char}(k)=3$, and $\operatorname{dim} W=7$ or 8 .

g) $H=\Omega_{8}^{ \pm}(2), \operatorname{char}(k)=3$.

The cases (c) and (e) are as in the statement of the result; we now show that in cases (a), (b), (d), (f) and (g), the assumption that $W$ is $Q$-linear large leads to a contradiction.

We first note that in cases (f) and (g), using the modular character tables in GAP08, it is straightforward to check that there is no $Q$-linear large representation for which the symmetric, alternating or tensor square is irreducible. 
In case $H=\mathbf{U}_{r+1}(2)$ the $Q$-linear large hypothesis implies that $r>5$. Now by Proposition 5.3, $\operatorname{dim} W \geq b_{2} \geq \beta:=2^{r}\left(2^{r-2}-1\right)\left(2^{r-1}-1\right) / 3$ and thus the upper bound for the dimension of $V$ given in (a) above is less than $\left(\begin{array}{c}\beta \\ 2\end{array}\right)-2$, and hence $\left.V\right|_{H}$ is reducible.

If $H$ is of type $\mathbf{O}_{n}$ or $\boldsymbol{\Omega}_{2 r}$ as in (b) or (d), then our estimate for $b_{2}$ (see Table 4) exceeds $\operatorname{dim} W$ or as above $\left(\begin{array}{c}b_{2} \\ 2\end{array}\right)-2$ exceeds the upper bound for $\operatorname{dim} V$, unless $H \in\left\{\mathbf{O}_{9}(2), \mathbf{O}_{7}(4)\right\}$, when $b_{2}$ was not defined. Thus, as above we conclude that either $\left.V\right|_{H}$ is reducible or $H$ is one of the groups $\mathbf{O}_{9}(2), \mathbf{O}_{7}(4)$. For the first of the two exceptional cases, we use GAP08 to check that the alternating square of the three lowest degree nontrivial characters is irreducible. However, these characters correspond to modules which do not satisfy the $Q$-linear large hypothesis. For the larger character degrees, we may argue as above to see that $\left.V\right|_{H}$ is reducible.

For $\mathbf{O}_{7}(4) \cong \mathbf{S}_{6}(4)$ the ordinary character table is in GAP08. The maximal degree of an irreducible character is 371280 and thus if $\left.V\right|_{H}$ is irreducible, $\operatorname{dim} W$ is bounded above by 862 . Now Table 2 shows that the possible orbit lengths of $L_{\lambda}$ are 255, 408 and 360, respectively, with $L_{\lambda}=4^{3}: \mathrm{Alt}_{5}, \mathbf{O}_{4}^{+}(4)$ and $\mathbf{O}_{4}^{-}(4)$, respectively. The argument on page 398 in the proof of [MMT02, 5.5] showing that since the representation affording $W$ has no root group in its kernel, the length of the orbit of $L_{\lambda}$ is 408 or 360, applies here. Now we note that the minimal dimension of an $\mathbf{O}_{4}^{+}(4)$-, respectively $\mathbf{O}_{4}^{-}(4)$-module, is at least 3 , which means that the dimension of a $Q$-linear large module is at least $360 \cdot 3>862$. Hence $\left.V\right|_{H}$ is reducible, as required.

Next we strengthen Proposition 5.7 of [MMT02] to eliminate possibility (c) in the list above.

Lemma 6.7. Let $H=\mathbf{O}_{2 r+1}(2), r \geq 4$, and $\operatorname{char}(k)=3$. If $W$ is a nontrivial irreducible $k H$ module and $V$ is a restricted irreducible $k G$-module with highest weight $\lambda$ satisfying $e(\lambda)=2$, and such that $\left.V\right|_{H}$ is irreducible, then

$$
\operatorname{dim} V \leq 2^{r-3}\left(2^{r-2}-1\right)\left(2^{2 r}-1\right)\left(2^{2 r-2}-1\right) .
$$

Moreover, if $W$ is a $Q$-linear large $k H$-module, then $\left.V\right|_{H}$ is reducible.

Proof. Let $P$ be the parabolic subgroup of $H$ which stabilizes a totally singular 2-space of the natural module of $H$. The unipotent radical $U$ of $P$ satisfies $[U, U]<Z(U)$ with $|[U, U]|=2$ and $|Z(U)|=8$. Now using the Chevalley commutator relations and the fact that the field of definition of $H$ is of even characteristic, we see that the subgroup of $U$ which is generated by the long root subgroups of $U$ is an extraspecial subgroup $E$ of $U$ of order $2^{1+2(2 r-4)}$. Moreover, the normalizer of $E$ in $P$ contains $U$ as well as $C:=S_{3} \times \Omega_{2 r-4}^{-}(2)$. Now, as was asserted in the proof of Proposition 5.5 of [MMT02, the orbits of $P$ on $Z(U)^{*}$ have lengths 1, 3 and 3. The character corresponding to the orbit of length 1 contains $[U, U]$ in its kernel. So as $U$ acts faithfully on $W$, it must be the case that $Z(U)$ acts on $W$ in such a way that at least one of the orbits of length 3 of $Z(U)^{*}$ is represented. Labelling the characters of such an orbit by $\chi_{i}, 1 \leq i \leq 3$, we then have $W_{\chi_{i}} \neq 0$. The weight spaces $W_{\chi_{i}}$ are equivalent $E C^{\infty}$-modules. Now Proposition 2.3 of MMT02 implies that $V$ contains a nontrivial $E C^{\infty}$-invariant vector $v$. (Note that as $q=2$, the $\chi_{i}$ are self-dual.) Now $k U C v$ is a $U C$-invariant submodule of $V$. Also $U C / E C^{\infty} \cong S_{4}$, so $k U C v$ contains a $U C$-invariant submodule of dimension at most 3 . Thus $\operatorname{dim} V$ is bounded above by

$$
3[H: U C]=2^{r-3}\left(2^{r-2}-1\right)\left(2^{2 r}-1\right)\left(2^{2 r-2}-1\right),
$$

which proves our first claim.

Now assume $W$ is $Q$-linear large and recall that $V$ is the maximal-dimensional composition factor of $\Lambda^{2}(W)$ or $S^{2}(W)$. When $r \geq 5$, Proposition 5.3 implies that $\operatorname{dim} W \geq b_{2} \geq$ $\left(2^{2 r-2}-1\right) 2^{r-2}\left(2^{r-1}-1\right)$ which implies that $\left(\begin{array}{c}\beta \\ 2\end{array}\right)-2$ is larger than $\operatorname{dim} V$. When $r=4$, the 3-modular character table of $H$ is available in GAP08 and we check directly that $H$ acts reducibly on $V$ whenever $W$ is $Q$-linear large. 
We now strengthen Proposition 5.13 of MMT02 to eliminate possibility (e) from the list of possible obstructions above.

Lemma 6.8. Let $H=\boldsymbol{\Omega}_{2 r}^{ \pm}(2), r \geq 5$, and $\operatorname{char}(k)=3$. If $W$ is a nontrivial irreducible $k H$ module and $V$ is a restricted irreducible $k G$-module with highest weight $\lambda$ satisfying $e(\lambda)=2$, and such that $\left.V\right|_{H}$ is irreducible, then

$$
\operatorname{dim} V \leq 2^{2 r-5}\left(2^{r} \mp 1\right)\left(2^{2 r-2}-1\right)\left(2^{r-2} \mp 1\right) .
$$

Moreover, if $W$ is Q-linear large, then $\left.V\right|_{H}$ is reducible.

Proof. Let $P \subset H$ be the stabilizer of a singular 1-space of the natural module of $H$ with unipotent radical $U$, and let $C=\Omega_{2}^{-}(2) \times \Omega_{2 r-4}^{\mp}(2) \cong S_{3} \times C^{\infty}$ be the naturally defined subgroup of the Levi factor $\Omega_{2 r-2}^{+}(2)$ of $P$.

Now the unipotent radical $U$ has the structure of a $C$-module, the sum of the natural module for $\Omega_{2}^{-}(2)$ and the natural module for $\Omega_{2 r-4}^{\mp}(2)$. In particular, there is a subgroup $K$ of $U$ corresponding to the first summand, which is an elementary abelian 2-group, containing $U C$ in its normalizer.

Now $\left.W\right|_{K}$ contains nontrivial weight spaces for each of the three distinct weights in $K^{*}=\operatorname{Hom}\left(K /[K, K], k^{*}\right)$. Again Proposition 2.3 of [MMT02 yields a 1-dimensional $U C^{\infty}$ _ submodule in $V$ and thus, as in the proof of the previous lemma, a 3-dimensional $U C$-invariant submodule of $V$. This yields

$$
\operatorname{dim} V \leq 3[H: U C]=2^{2 r-5}\left(2^{r} \mp 1\right)\left(2^{2 r-2}-1\right)\left(2^{r-2} \mp 1\right),
$$

which is our claim.

Now assume $W$ is $Q$-linear large and recall that $V$ is the maximal-dimensional composition factor of $\Lambda^{2}(W)$ or $S^{2}(W)$. Note that when $r \geq 7, \operatorname{dim} W \geq b_{2} \geq \beta:=\left(2 \frac{2^{2 r-6}-1}{3}-2^{r-3}-\right.$ $r+4)\left(2^{2 r-3}-2^{r-1}+2^{r-2}-1\right)$ and again $\left(\begin{array}{c}\beta \\ 2\end{array}\right)-2$ is larger than $\operatorname{dim} V$, when $r \geq 7$. When $r=5$, we consult the 3-modular character tables in GAP08] and then, as above, we check our assertion directly.

When $r=6$, then our bound for $\operatorname{dim} V$ yields that $\operatorname{dim} W \leq 17011$. Since $O^{p}\left(L_{\chi}^{\infty}\right) \supset$ $\Omega_{8}^{+}(2)$, we see that $\operatorname{dim} W \geq 495 \cdot b$, where $b$ is the minimal dimension of an irreducible $k \Omega_{8}^{+}(2)$-module. If this dimension is at least 35 , we have the desired contradiction. The only other possibility is a 28 -dimensional $k \Omega_{8}^{+}(2)$-module. More precisely, we have to analyze the situation where $H=\Omega_{12}^{+}(2),\left[L: L_{\chi}\right]=2^{9}+32-16-1=527$ and the socle of $W_{\chi}$ contains the 28-dimensional module for $2^{8}: \boldsymbol{\Omega}_{8}^{+}(2)$; call this submodule $X \subset \operatorname{soc}\left(W_{\chi} \mid L_{\chi}\right)$. As $q=2$, the group $Q$ acts trivially on the alternating and symmetric squares of $W_{\chi}$.

The alternating and symmetric squares of $X$ possess 28-, respectively 35-dimensional submodules. Thus the group $Q: 2^{8}: \boldsymbol{\Omega}_{8}^{+}(2)$ has a 28 -, respectively 35 -dimensional submodule on $V$. Consequently $\operatorname{dim} V$ is bounded above by $28 \cdot 527 \cdot\left(2^{11}+2^{6}-2^{5}-1\right)\left(=28 \cdot\left[H: Q L_{\chi}\right]\right)$, respectively $35 \cdot 527 \cdot 2079$. But these numbers are smaller than $\left(\begin{array}{c}28.527 \\ 2\end{array}\right)-2$, respectively $\left(\begin{array}{c}35 \cdot 527+1 \\ 2\end{array}\right)-2$. So again we have our usual contradiction.

We are now ready to establish the main result of this section:

Theorem 6.9. Let $W$ be a $Q$-linear large $k H$-module, $G \subset \mathrm{GL}(W)$ the smallest classical group containing the image of $H$ under the associated representation, and let $V$ be an irreducible $k G$-module with restricted highest weight $\lambda$. Then either $V$ is isomorphic to $W$ or $W^{*}$ or $\left.V\right|_{H}$ is reducible.

Proof. Up to Lemma 6.5, we did not require the $k H$-module $W$ to be irreducible. Moreover, Proposition 6.3 and Lemma 6.5 show that if $W$ is a $Q$-linear large $k H$-module, then $\left.V\right|_{H}$ is reducible unless $e(\lambda) \leq 2$. If $e(\lambda)=2$, then $V$ is the heart of the symmetric or alternating 
square of the natural $k G$-module $W$. But then it is clear that if $W$ is a reducible $k H$-module, the heart of the alternating or symmetric square is again reducible as a $k H$-module. Hence, the irreducibility of $\left.V\right|_{H}$ implies that $\left.W\right|_{H}$ is irreducible and then the result follows from the preceding two lemmas.

\section{The nonrestricted case}

In this section we assume that $W$ is a $Q$-linear large $k H$-module, as usual, $G \subset \mathrm{SL}(W)$ is the smallest classical group containing the image of $H$ under the corresponding representation, and $V$ is an irreducible $k G$-module with highest weight $\lambda$. Moreover, we assume that $V$ is not a Frobenius twist of $W$ or $W^{*}$. Having treated the case where $\lambda$ is restricted we now turn to the case where the highest weight $\lambda$ is not restricted. This hypothesis, together with Steinberg's tensor product theorem, implies that $V$ decomposes as a twisted tensor product. Write $\lambda=\sum_{i=0}^{t}(\operatorname{char}(k))^{i} \mu_{i}$, where the $\mu_{i}$, for $0 \leq i \leq t$, are restricted highest weights, and let $F$ denote the standard Frobenius morphism of $G \subset \mathrm{GL}(W)$. If exactly one of the $\mu_{i}$ is nonzero, then $H$ acts irreducibly on $V$ if and only if $H$ acts irreducibly on the irreducible $k G$-module with highest weight $\mu_{i}$. Since there are no such configurations, by Theorem 6.9. we assume that at least 2 of the $\mu_{i}$ are nonzero.

Lemma 7.1. If $\lambda=\sum_{i=0}^{t}(\operatorname{char}(k))^{i} \mu_{i}$, and $e\left(\mu_{i}\right)>1$ for some $i$, then $\left.V\right|_{H}$ is reducible.

Proof. Our hypothesis on $\lambda$ implies that $V=V\left(\mu_{0}\right) \otimes V\left(\mu_{1}\right)^{F} \otimes \cdots \otimes V\left(\mu_{t}\right)^{F^{t}}$, where $V\left(\mu_{i}\right)$ is a restricted highest weight module for $G$. If $e\left(\mu_{i}\right)>1$ then Theorem 6.9 implies that $\left.V\left(\mu_{i}\right)\right|_{H}$ is reducible. As the tensor product is distributive our claim follows.

So without loss of generality, we now assume that each nontrivial tensor factor of $V$ is a Frobenius twist of $W$ or $W^{*}$.

Proposition 7.2. Assume $H$ is of type $\mathbf{L}$, and $V$ is an irreducible $k G$-module with highest weight $\lambda=\sum_{i=0}^{t}(\operatorname{char}(k))^{i} \mu_{i}$. If $\mu_{i} \neq 0 \neq \mu_{j}$ for some $0 \leq i \neq j \leq t$, then $\left.V\right|_{H}$ is reducible.

Proof. Suppose the contrary. Then Lemma 7.1 implies that $V$ is a tensor product of $e$ Frobenius twists of restricted $k G$-modules, each of which is isomorphic to either $W$ or $W^{*}$, where $2 \leq e \leq t+1$. If $H=\mathbf{L}_{r+1}(q)$ with $q \geq 4$ and $r \geq 1$, then [MT01, 3.2] implies that $\left.V\right|_{H}$ is reducible. If $H=\mathbf{L}_{r+1}(3)$, with $r \geq 2$, then [MT01, 3.4] implies that the tensor product of two $k H$-modules is reducible unless one of the factors is a Weil module. It follows from the basic properties of Weil modules (see GT99]) that if $W$ is $Q$-linear large then none of $W$, $W^{*}$, or any Frobenius twists of these modules are Weil modules.

Hence, by Lemma 5.1 and the above considerations, we are reduced to the case where $H=\mathbf{L}_{r+1}(2)$ with $r \geq 4$. We now refer to [MT01, 3.3] to see that the tensor product of any two non-trivial $k H$-modules is reducible, thus establishing the proposition.

Let $P<H$ be as in Sections 1 and 5 , the stabilizer of a singular 1-space of the natural module for $H$. Write as usual $P=Q L$, where $Q=O_{p}(P)$. Using Table 2, we see that $L$ has up to three orbits $\mathcal{O}_{i}, 1 \leq i \leq 3$ on the non-trivial linear characters of $Q$. Define $W_{\mathcal{O}_{i}}:=\oplus_{\lambda \in \mathcal{O}_{i}} W_{\lambda}$ and note that $W_{\mathcal{O}_{i}}$ is a $P$-module. For a $k$-subspace $U$ of $W$, we write $U^{F}$ for the image of $U$ under the semilinear map induced by $F$.

Lemma 7.3. With the notation as above we have that $W_{\mathcal{O}_{i}}$ and $\left(W_{\mathcal{O}_{i}}\right)^{F}$ are equivalent $k Q$ modules for each $i$.

Proof. The $k Q$-module $W_{\mathcal{O}_{i}}$ is a direct sum of $\left|\mathcal{O}_{i}\right|$ isotypic components. As $F$ is semilinear we see that $W_{\mathcal{O}_{i}}^{F}$ is also a direct sum of $\left|\mathcal{O}_{i}\right|$ isotypic components. Now, using Table 2, we observe that the $P$-orbits on $\operatorname{Hom}\left(Q /[Q, Q], k^{*}\right)$ have pairwise distinct lengths, and thus $W_{\mathcal{O}_{i}}$ and $\left(W_{\mathcal{O}_{i}}\right)^{F}$ are equivalent $k Q$-modules. 
The proof of the lemma shows that for $\chi \in \operatorname{Hom}\left(Q /[Q, Q], k^{*}\right)$, with $W_{\chi} \neq 0, F$ can be modified (multiplied) by an element of $P$ to guarantee that $W_{\chi}$ is $F$-invariant. Henceforth we assume this is the case. (Of course the element of $P$ needed to guarantee this is not independent of our choice of $\chi$.)

Lemma 7.4. If $\chi$ is a linear character of $Q$ and $S \subset W_{\chi}$ is an $L_{\chi}$-invariant $k$-subspace, then we can choose $F$ such that $W_{\chi}=\left(W^{F}\right)_{\chi}$ and $S^{F}$ is an $F\left(L_{\chi}\right)$-invariant $k$-subspace.

Proof. We choose $F$ as discussed above so that $W_{\chi}$ is $F$-invariant. The second assertion follows from the fact that semilinear maps preserve isomorphisms and centralizers; that is, $F\left(C_{\mathrm{GL}\left(W_{\chi}\right)}\left(L_{\chi}\right)\right)=C_{\mathrm{GL}\left(W_{\chi}\right)}\left(F\left(L_{\chi}\right)\right)$ and hence the endomorphism algebras of $\left.W_{\chi}\right|_{L_{\chi}}$ and of $\left.W_{\chi}\right|_{F\left(L_{\chi}\right)}$ are mapped isomorphically one onto the other and in particular the fields of definition are preserved.

Lemma 7.5. Assume $W$ is an irreducible $Q$-linear large module for $H$. If $H$ is not of type $\mathbf{L}$, $\lambda=\sum_{i=0}^{t}(\operatorname{char}(k))^{i} \mu_{i}$, and $\left.V\right|_{H}$ is irreducible, then the number of non-trivial tensor factors is at most two, and moreover, the $k H$-modules $W$ and $W^{F}$ are inequivalent.

Proof. As in the proof of Proposition 7.2, we may assume that $V$ is a tensor product of $e$ Frobenius twists of restricted irreducible $k G$-modules each of which is isomorphic to one of $W$ and $W^{*}$, and where $2 \leq e \leq t+1$. Now $F$ acts semilinearly on $W$ and is a bijective morphism of the group $G$. Hence, $F^{i}(H)$ is a subgroup of $G$ acting irreducibly on $W^{F^{i}}$ and on $\left(W^{*}\right)^{F^{i}}$, for all $i \geq 0$. If $W$ and $W^{F}$ are equivalent $k H$-modules, then any tensor product involving Frobenius twists of $W$ and $W^{*}$ must necessarily be reducible; hence $W$ and $W^{F}$ are inequivalent $k H$-modules.

We now argue as in the proofs of Lemma 6.2 and Proposition 6.3 to produce a subspace of $V$ which is invariant under $P_{\chi}=Q L_{\chi}$ for some linear character $\chi \in Q^{*}$ such that $W_{\chi} \neq 0$. As usual we let $S \subset W_{\chi}$ be an irreducible $P_{\chi}$-submodule. Then the $P_{\chi}$-module $W^{F}$ has a submodule $S^{F}$ of dimension equal to $\operatorname{dim} S$. By Lemma 7.4 all tensor factors of $V$ possess a $P_{\chi}$-invariant submodule of dimension equal to $\operatorname{dim} S$. This yields an upper bound for $\operatorname{dim}(V)$ namely

$$
\operatorname{dim} V \leq(\operatorname{dim} S)^{e}\left[H: P_{\chi}\right]=(\operatorname{dim} S)^{e}[H: P]\left[L: L_{\chi}\right]
$$

Clearly, $\operatorname{dim} W \geq(\operatorname{dim} S)\left[P: P_{\chi}\right]=(\operatorname{dim} S)\left[L: L_{\chi}\right]$, which yields a lower bound for $\operatorname{dim} V$, and we therefore have the following two inequalities:

$$
(\operatorname{dim} S)^{e}\left[L: L_{\chi}\right]^{e} \leq \operatorname{dim} V \leq(\operatorname{dim} S)^{e}[H: P]\left[L: L_{\chi}\right]
$$

In the proof of Proposition 6.3 we checked that

$$
\left(\begin{array}{c}
{\left[L: L_{\chi}\right]-1} \\
3
\end{array}\right)>\left[H: P_{\chi}\right]
$$

for all $H \neq \mathbf{O}_{7}(2), \boldsymbol{\Omega}_{8}^{+}(2), \mathbf{O}_{9}(2)$. As $\left(\begin{array}{c}{\left[L: L_{\chi}\right]-1} \\ 3\end{array}\right)<\left[L: L_{\chi}\right]^{3}$, we have that $e \leq 2$ for all $H \neq \mathbf{O}_{7}(2), \boldsymbol{\Omega}_{8}^{+}(2), \mathbf{O}_{9}(2)$.

If $H=\mathbf{O}_{7}(2)$, then arguing as in the proof of Lemma 6.5, we see that $\operatorname{dim} V \leq 720$. Since $\sqrt[3]{720}<9$ and any $Q$-linear large module for $H$ has dimension at least 14, $e$ can be at most 2. For $H=\Omega_{8}^{+}(2)$, we have $\operatorname{dim} V \leq 6075$, while $\operatorname{dim} W \geq 104$, and again we have $e \leq 2$. Finally, if $H=\mathbf{O}_{9}(2)$, then we check that $\operatorname{dim} W \geq 28 \cdot 4=112$ and thus $(\operatorname{dim} W)^{3}$ exceeds the degree of any irreducible $k H$-representation, completing the proof that $e \leq 2$.

Proposition 7.6. If $H$ is not of type $\mathbf{L}$ and $V=W \otimes W^{F^{i}}$ or $V=W \otimes\left(W^{*}\right)^{F^{i}}$, then $\left.V\right|_{H}$ is reducible unless:

1. $q \leq 3$, or 
2. $q$ is even and $H=\mathbf{O}_{2 r+1}(q)$.

Proof. The content of Theorem 1.1 of [MT01] is that $H=X_{r}(q)$ acts reducibly on tensor products of irreducible cross-characteristic $H$-modules unless one of the following holds:

- $q \leq 3$

- $H=\emptyset_{2 r+1}(q)$ with $q$-even;

- $H=\mathbf{S}_{2 r}(5)$; or

- $H=\mathbf{L}_{3}(4)$.

The last case is ruled out by Lemma 5.1. The case $H=\mathbf{S}_{2 r}(5)$ can be eliminated using Proposition 5.2 (ii) of [MT01], as our hypothesis that $W$ is $Q$-linear large implies that $W$ is not a Weil module.

To complete the proof of Theorem 1, we must handle case 2 . of the previous result in case $q \geq 4$. This is covered by the following:

Proposition 7.7. If $q \geq 4$ is even, $H=\mathbf{O}_{2 r+1}(q)$ with $r \geq 3, W$ is an absolutely irreducible $k H$-module, and $V=W \otimes W^{F^{i}}$ or $V=W \otimes\left(W^{*}\right)^{F^{i}}$, then $\left.V\right|_{H}$ is reducible.

Before beginning the proof of the proposition, we set up some further notation and establish 2 lemmas.

Let $P_{2}$ denote the stabilizer in $H$ of a singular 2-space. We denote the unipotent radical of $P_{2}$ by $Q_{2}$ and fix a Levi factor $L_{2}$, so $L_{2} \cong \mathrm{GL}_{2}(q) \mathrm{O}_{2 r-3}(q)$. Now $Z\left(Q_{2}\right)$ is an elementary abelian group of order $q^{3}$, the natural module of the $O_{3}(q) \cong \mathrm{PGL}_{2}(q)$ subgroup of $L_{2}$. By $W_{\lambda}$ we denote the $Z\left(Q_{2}\right)$ weight spaces of $W$, for $\lambda \in \operatorname{Hom}\left(Z\left(Q_{2}\right), k^{*}\right)$; hereafter, write $Z\left(Q_{2}\right)^{*}$ for this last Hom space. The group $L_{2}$ has three orbits on the non-trivial characters of $Z\left(Q_{2}\right)$ and the results of Table 2 show that at least $q(q-1)^{2} / 2$ of the weight spaces are non-trivial.

As the $L_{2}$-orbits on $Z\left(Q_{2}\right)^{*}$ have distinct lengths, we can argue as in the proof of Lemma7.3 to obtain:

Lemma 7.8. Denote by $\mathcal{O}_{i}$, for $i \in I$, the orbits of $L_{2}$ on $Z\left(Q_{2}\right)^{*}$. We have that $W_{\mathcal{O}_{i}}$ and $\left(W_{\mathcal{O}_{i}}\right)^{F}$ are equivalent $k Q_{2}$-modules for all $i$.

Now we require some information about the irreducible characters of $Q_{2}$ and the construction of those which are nonlinear.

Lemma 7.9. The irreducible characters of $Q_{2}$ are either

- linear, and there are $\left|Q_{2}\right| / q$ of them, or

- of degree $\left[Q_{2}: Z\left(Q_{2}\right)\right]^{1 / 2}$, and there are $q^{3}-q^{2}$ of them.

Proof. The commutator subgroup of $Q_{2}$ has order $q$ so the statement concerning linear characters is clear. Let $R_{2}$ be the subgroup of $Q_{2}$ generated by the long root subgroups (with respect to a fixed torus inside $\left.P_{2}\right)$. Note that $\left[R_{2}, R_{2}\right]=\left[Q_{2}, Q_{2}\right]$ and hence $Z\left(Q_{2}\right)$ has exactly $q^{3}-q^{2}$ characters which do not have $\left[R_{2}, R_{2}\right]$ in their kernel. Moreover, we note that the group $R_{2}$ is special, and such that $\left[x, R_{2}\right]=Z\left(R_{2}\right)$ for all $x \in R_{2} \backslash Z\left(R_{2}\right)$. Thus all nonlinear irreducible characters of $R_{2}$ have degree $\left[R_{2}: Z\left(R_{2}\right)\right]^{1 / 2}=\left[Q_{2}: Z\left(Q_{2}\right)\right]^{1 / 2}$.

Now let $\lambda$ be a character of $Z\left(Q_{2}\right)$ which does not have $\left[R_{2}, R_{2}\right]$ in its kernel. Let $A$ be an abelian subgroup of $R_{2}$ such that $\left\langle A, Z\left(R_{2}\right)\right\rangle$ is a maximal abelian subgroup of $R_{2}$. Since $A \cap Z\left(R_{2}\right)=1$, and $Z\left(Q_{2}\right) \cap R_{2}=Z\left(R_{2}\right)$, we have that $A \cap Z\left(Q_{2}\right)=1$ as well. As $\left\langle A, Z\left(Q_{2}\right)\right\rangle$ is an abelian subgroup of $Q_{2}$ we see that $\lambda$ extends to a linear character $\tilde{\lambda}$ of $\left\langle A, Z\left(Q_{2}\right)\right\rangle$. Thus the degree of $\tilde{\lambda} \uparrow Q_{2}$ is $\left[Q_{2}: Z\left(Q_{2}\right)\right]^{1 / 2}$. The restriction of $\tilde{\lambda} \uparrow Q_{2}$ to $Z\left(R_{2}\right)$ is 
nontrivial and again since $R_{2}$ is special, $\tilde{\lambda} \uparrow^{Q_{2}} \downarrow_{R_{2}}$ is irreducible; hence $\tilde{\lambda} \uparrow Q_{2}$ is an irreducible character of degree claimed above. Now the construction of $\tilde{\lambda} \uparrow Q_{2}$ can be carried out for all characters of $Z\left(Q_{2}\right)$ which do not have $\left[R_{2}, R_{2}\right]$ in their kernel. Evidently all such characters are pairwise distinct as their restrictions to $Z\left(Q_{2}\right)$ are distinct as well. So summing the squares of the degrees of the irreducible $Q_{2}$ characters constructed so far yields $\left|Q_{2}\right|$. Thus the first orthogonality relation of characters shows that we have constructed all irreducible characters of $Q_{2}$.

Proof of Proposition 7.7. Arguing as in the proof of Lemma 7.4 and using Lemma 7.8, we have that $W_{\lambda}$ and $\left(W^{F}\right)_{\lambda}$ are equivalent as $Q_{2}$-modules. We note here that $\operatorname{dim} W_{\lambda}=m_{\lambda}\left[Q_{2}\right.$ : $\left.Z\left(Q_{2}\right)\right]^{1 / 2}$, where $m_{\lambda}$ is the multiplicity of the $Q_{2}$ character $\tilde{\lambda} \uparrow Q_{2}$ in $W_{\lambda}$.

This leads to a lower bound

$$
\operatorname{dim} V \geq\left(m_{\lambda}\right)^{2}\left[Q_{2}: Z\left(Q_{2}\right)\right]\left(q^{2}-q\right)^{2}(q-1)^{2} / 4
$$

We now derive an upper bound for $\operatorname{dim} V$. Recall from above that the subgroup $R_{2}$ of $Q_{2}$ generated by long root elements is special and that any irreducible representation of $Q_{2}$ which restricts nontrivially to $Z\left(R_{2}\right)$ restricts irreducibly to $R_{2}$. As $q$ is even, if $\lambda \in Z\left(Q_{2}\right)^{*}$ and $Z\left(R_{2}\right) \not \subset \operatorname{Ker}(\lambda)$, then $Z\left(Q_{2}\right)$ acts as minus the identity on $W_{\lambda}$ and thus acts trivially on $W_{\lambda} \otimes W_{\lambda}^{F}$. Thus the action of $Q_{2}$ on $W_{\lambda} \otimes W_{\lambda}^{F}$ is $m_{\lambda}^{2}$ times the regular character of $Q_{2} / Z\left(Q_{2}\right)$. In particular, $C_{W_{\lambda} \otimes W_{\lambda}^{F}}\left(Q_{2}\right) \neq 0$ and this subspace contains a $Q_{2} \mathrm{O}_{2 r-3}(q)$-submodule $S_{\lambda}$ of dimension at most $\left(m_{\lambda}\right)^{2}$. Thus we see that the $P_{2}$-module $\sum_{\lambda} C_{W_{\lambda} \otimes W_{\lambda}^{F}}\left(Q_{2}\right)$, on which $Q_{2}$ acts trivially, affords a $\mathrm{GL}_{2}(q) \times \mathrm{O}_{2 r-3}(q)$ module. As the $\mathrm{O}_{2 r-3}(q)$-submodule $S_{\lambda}$ is contained in $\sum_{\lambda} C_{W_{\lambda} \otimes W_{\lambda}^{F}}\left(Q_{2}\right)$, we see by Frobenius reciprocity that $\sum_{\lambda} C_{W_{\lambda} \otimes W_{\lambda}^{F}}\left(Q_{2}\right)$ contains a quotient of the $P_{2}$-module $S_{\lambda} \uparrow_{Q_{2}}^{P_{2}} \mathrm{O}_{2 r-3}(q)$, whose socle contains a nontrivial irreducible $\mathrm{GL}_{2}(q) \times \mathrm{O}_{2 r-3}$ submodule $S$. As $\operatorname{dim} S \leq(q+1) m_{\lambda}^{2}$, we see that

$$
\begin{gathered}
\operatorname{dim} V \leq(q+1) m_{\lambda}^{2}\left[H: P_{2}\right]=(q+1) m_{\lambda}^{2}\left(q^{2 r}-1\right)\left(q^{2 r-2}-1\right) /\left(q^{2}-1\right)(q-1) \\
=m_{\lambda}^{2}\left(q^{2 r}-1\right)\left(q^{2 r-2}-1\right) /(q-1)^{2} .
\end{gathered}
$$

Combining the upper and lower bounds and using the fact that $\left[Q_{2}: Z\left(Q_{2}\right)\right]=q^{2(2 r-4)}$, leads to the inequality

$$
q^{2(2 r-4)}\left(q^{2}-q\right)^{2}(q-1)^{2}(q-1)^{2}<4\left(q^{2 r}-1\right)\left(q^{2 r-2}-1\right),
$$

which reduces to

$$
q^{(4 r-6)}(q-1)^{6}<4\left(q^{2 r}-1\right)\left(q^{2 r-2}-1\right),
$$

and leads to a contradiction when $q \geq 8$ and $r \geq 3$.

When $q=4$ we improve our upper bound for $\operatorname{dim} V$ by $1 / 2$ using induction. All the modular character tables for $\mathrm{O}_{3}(4) \cong \mathrm{SL}_{2}(4)$ and $\mathrm{O}_{5}(4) \cong \mathrm{Sp}_{4}(4)$ are in GAP08] and we see that $W \otimes W^{\prime}$ is reducible, for any pair of irreducible modules $W$ and $W^{\prime}$, and so in particular, $W \otimes W^{F}$ is reducible. Thus, by dualizing if necessary, we see that $W_{\lambda} \otimes\left(W^{F}\right)_{\lambda}$ has an $\mathrm{O}_{2 r-3}(4)$-submodule of dimension at most $m_{\lambda}^{2} / 2$ (rather than $m_{\lambda}^{2}$ as before), which leads to the improved upper bound. The improved inequality yields a contradiction when $q=4$.

This completes the proof of the proposition.

We have now established:

Theorem 7.10. Let $W$ be an irreducible $Q$-linear large $k H$-module, $G \subset \mathrm{GL}(W)$ the smallest classical group containing the image of $H$, and $V$ a tensor decomposable non-restricted irreducible $k G$-module. Let $F$ be a fixed char $(k)$-power Frobenius morphism of the group $G$. If $V$ is a twisted tensor product of restricted irreducible $k G$-modules, then $\left.V\right|_{H}$ is reducible unless $q \leq 3$ and $V$ is a Frobenius twist of a module of the form $X \otimes Y^{F}$, where $X, Y \in\left\{W, W^{*}\right\}$, and $Y^{F}$ is a Frobenius twist of $Y$ such that $\left.X\right|_{H}$ and $\left.Y^{F}\right|_{H}$ are inequivalent $k H$-modules. 


\section{References}

[Asc84] M. Aschbacher. On the maximal subgroups of the finite classical groups. Invent. Math., 76(3):469-514, 1984.

[BO98] C. Bessenrodt and J. B. Olsson. Branching of modular representations of the alternating groups. J. Algebra, 209(1):143-174, 1998.

[Bou68] N. Bourbaki. Éléments de mathématique. Fasc. XXXIV. Groupes et algèbres de Lie. Chapitre IV: Groupes de Coxeter et systèmes de Tits. Chapitre V: Groupes engendrés par des réflexions. Chapitre VI: systèmes de racines. Actualités Scientifiques et Industrielles, No. 1337. Hermann, Paris, 1968.

[Bra] J. Bray. Overgroups of $C_{6}$ type groups. Preprint.

[BK01] J. Brundan and A. S. Kleshchev. Representations of the symmetric group which are irreducible over subgroups. J. Reine Angew. Math., 530:145-190, 2001.

[Car89] R. W. Carter. Simple groups of Lie type. Wiley Classics Library. John Wiley \& Sons Inc., New York, 1989. Reprint of the 1972 original, A Wiley-Interscience Publication.

[CCN+85] J. H. Conway, R. T. Curtis, S .P. Norton, R. A. Parker, and R. A. Wilson. Atlas of finite groups. Oxford University Press, 1985.

[GAP08] The GAP Group. GAP - Groups, Algorithms, and Programming, Version 4.4.12, 2008.

[Gro02] L. C. Grove. Classical groups and geometric algebra, volume 39 of Graduate Studies in Mathematics. American Mathematical Society, Providence, RI, 2002.

[GMST02] R. M. Guralnick, K. Magaard, J. Saxl, and P. H. Tiep. Cross characteristic representations of symplectic and unitary groups. J. Algebra, 257(2):291-347, 2002.

[GT99] R. M. Guralnick and P. H. Tiep. Low-dimensional representations of special linear groups in cross characteristics. Proc. London Math. Soc. (3), 78(1):116-138, 1999.

[GT05] R. M. Guralnick and P. H. Tiep. Decompositions of small tensor powers and Larsen's conjecture. Represent. Theory, 9:138-208 (electronic), 2005.

[GT08] R. M. Guralnick and P. H. Tiep. Symmetric powers and a problem of Kollár and Larsen. Invent. Math., 174(3):505-554, 2008.

[HHM] G. Hiss, W. Husen, and K. Magaard. Irreducible and imprimitive modules for quasisimple groups I. Preprint.

[JS92] J. C. Jantzen and G. M. Seitz. On the representation theory of the symmetric groups. Proc. London Math. Soc. (3), 65(3):475-504, 1992.

[KS02] A. S. Kleshchev and J. Sheth. Representations of the alternating group which are irreducible over subgroups. Proc. London Math. Soc. (3), 84(1):194-212, 2002.

[KT04] A. S. Kleshchev and P. H. Tiep. On restrictions of modular spin representations of symmetric and alternating groups. Trans. Amer. Math. Soc., 356(5):1971-1999 (electronic), 2004.

[LS74] V. Landazuri and G. M. Seitz. On the minimal degrees of projective representations of the finite Chevalley groups. J. Algebra, 32:418-443, 1974. 
[Lüb01] F. Lübeck. Small degree representations of finite Chevalley groups in defining characteristic. LMS J. Comput. Math., 4:135-169 (electronic), 2001.

[MM98] K. Magaard and G. Malle. Irreducibility of alternating and symmetric squares. Manuscripta Math., 95(2):169-180, 1998.

[MMT02] K. Magaard, G. Malle, and P. H. Tiep. Irreducibility of tensor squares, symmetric squares and alternating squares. Pacific J. Math., 202(2):379-427, 2002.

[MT01] K. Magaard and P. H. Tiep. Irreducible tensor products of representations of finite quasi-simple groups of Lie type. In Modular representation theory of finite groups (Charlottesville, VA, 1998), pages 239-262. de Gruyter, Berlin, 2001.

[MT] K. Magaard and P. H. Tiep. Maximal subgroups of groups in classical groups which contain maximal members of $C_{6}$ or $C_{7}$. Preprint.

[Mal99] G. Malle. Almost irreducible tensor squares. Comm. Algebra, 27(3):1033-1051, 1999.

[Pre87] A. A. Premet. Weights of infinitesimally irreducible representations of Chevalley groups over a field of prime characteristic. Mat. Sb. (N.S.), 133(175)(2):167-183, $271,1987$.

[Sei87] G. M. Seitz. The maximal subgroups of classical algebraic groups. Mem. Amer. Math. Soc., 67(365):iv+286, 1987.

[Sei90] G. M. Seitz. Cross-characteristic embeddings of finite groups of Lie type. Proc. London Math. Soc. (3), 60(1):166-200, 1990.

[SZ93] G. M. Seitz and A. E. Zalesskii. On the minimal degrees of projective representations of the finite Chevalley groups. II. J. Algebra, 158(1):233-243, 1993.

[Smi82] S. D. Smith. Irreducible modules and parabolic subgroups. J. Algebra, 75(1):286$289,1982$. 